\title{
Criticality and correlated dynamics at the irreversibility transition in periodically driven colloidal suspensions
}

\author{
Elsen Tjhung \& Ludovic Berthier \\ Laboratoire Charles Coulomb, UMR 5221, CNRS and Université Montpellier, \\ Montpellier, France
}

\begin{abstract}
One possible framework to interpret the irreversibility transition observed in periodically driven colloidal suspensions is that of a non-equilibrium phase transition towards an absorbing reversible state at low amplitude of the driving force. We consider a simple numerical model for driven suspensions which allows us to characterize in great detail a large body of physical observables that can be experimentally determined to assess the existence and universality class of such a non-equilibrium phase transition. Characterizing the behaviour of static and dynamic correlation functions both in real and Fourier space we determine in particular several critical exponents for our model, which take values that are in good agreement with the universality class of directed percolation. We also provide a detailed analysis of single-particle and collective dynamics of the system near the phase transition, which appear intermittent and spatially correlated over diverging timescales and lengthscales, and provide clear signatures of the underlying criticality.
\end{abstract}

\section{Introduction}

Non-equilibrium phase transitions have been gaining interests in recent years [1, 2, 3]. Whereas theoretical models can be organised in well-studied universality classes, the interpretation of experimental realisations is always more challenging, due to the inherent complexity of an experimental set-up compared to the simplicity of theoretical models. In this respect, the experimental study by Pine and coworkers [4, 5, 6] of a relatively simple driven suspension of non-Brownian particles displaying an apparent continuous phase transition towards an absorbing state offered a very promising new path for studying experimentally non-equilibrium phase transitions [7, 8, 9, 10, 11. In the original work [4, the authors studied a colloidal suspension driven out of equilibrium by shearing the system periodically with some frequency $\omega_{0}$ and a maximum shear amplitude $a$. It has been found that below some critical shear amplitude $a_{c}$ (which depends on the volume fraction $\phi$ ), the system evolves into an absorbing state in which all particles move reversibly in response to the external periodic shear. In other words, when observed stroboscopically, single-particle motion appears to be frozen and thus the diffusion constant $D$ is zero. On the other hand above the critical shear amplitude 
$a_{c}$, the particle motion becomes irreversible and appears diffusive at long times from stroboscopic images. This qualitative transformation was suggested to correspond to an nonequilibrium phase transition from an absorbing reversible state with $D=0$ to an irreversible diffusive phase with $D>0$, and the results were compatible with a continuous phase transition [4, 5]. Interestingly at high density (not considered in this paper), such transitions have also been suggested to be associated with plastic yielding [8, 12, 13, 14].

Crucially, these colloidal particles are suspended in a very viscous solvent such that thermal effect is negligible over the duration of the experiment. The origin of irreversibility was, in fact, attributed to collisions between the particles provoked by the shear flow [5]. From this interpretation, Corté et al. then constructed a simple numerical model for their experiment on sheared non-Brownian suspensions, where microscopic irreversibility upon collisions in the real system is converted into a stochastic rule for binary collisions in the theoretical model [5]. (Long range hydrodynamic interactions were shown to play little role [15, 16].) However, it has also been suggested by several authors [7, 17, 18, 19, 20] that the onset of irreversibility could be due to chaotic motion of the particles rather than a nonequilibrium phase transition. In other words, macroscopic irreversibility could arise when the Lyapunov exponent is larger than some threshold without any diverging lengthscales or timescales. Therefore further experimental studies are necessary to show if there is, in fact, any diverging lengthscale in such systems. While both scenario may coexist in the original experiment, we have recently suggested a number of possible experimental measurements that should be able to determine the main source of the irreversibility transition observed experimentally [21]. We give here an extended account of these numerical findings.

The experimental suggestions we made were based on the demonstration that a simpler version of the model by Corté et al. displays a large number of experimentally accessible signatures of the criticality associated to the phase transition to an absorbing state. In the present paper, we provide a very extensive numerical study of this simplified model, which differs from the original one by the fact that stochastic rules for binary collisions are made isotropic [21, 22]. This small change in the definition allows us to characterise more accurately the critical properties of the system, which are not yet fully resolved. It has been initially suggested by Menon and Ramaswamy [23] that such periodically driven systems may belong to the universality class of conserved directed percolation (CDP). The conserved quantity, in this case, refers to the total number of particles. However up to date, there has been no accurate measurements of the critical exponents which may suggest a CDP universality class. For instance, the critical exponent for the order parameter $\beta$ is defined to be: $D \sim\left(a-a_{c}\right)^{\beta}$ as $a \rightarrow a_{c}^{+}$. Different values of $\beta$ have been reported in both experimental and numerical studies of bidimensional periodically sheared suspensions: $\beta=0.45 \pm 0.02$ [5], $\beta=0.67 \pm 0.09$ [8], $\beta=0.75 \pm 0.02$ [22], $\beta=0.59$ [24], $\beta=0.6 \pm 0.06$ [25] and $\beta=0.69 \pm 0.11$ [26, 27]. However from these values of $\beta$, we are still unable to determine if the system belongs to the conserved directed percolation (CDP) or directed percolation (DP) universality 
class. The value of $\beta$ in DP is 0.58 and in CDP is 0.64 for $2 \mathrm{D}$ systems [2]. The first aim of the present paper is to accurately measure $\beta$ as well as other critical exponents discussed below, to more precisely characterize the universality class of the model.

Another frequently measured quantity is a timescale $\tau$ which diverges close to criticality with a power law: $\tau \sim\left(a-a_{c}\right)^{-\nu_{\|}}$. The timescale $\tau$, for instance, can be defined as the timescale for the system to reach a steady state [5, 8, 25]. This timescale has been measured in the literature giving varying values of the critical exponents: $\nu_{\|}=1.33 \pm 0.02$ [5], $\nu_{\|}=1.1 \pm 0.3$ [8], $\nu_{\|}=1.30 \pm 0.06$ [22], and $\nu_{\|} \simeq 1.36 \pm 0.06$ [25] (all are in 2D). In this paper, we shall introduce four other ways of measuring $\tau$ independently. In particular, we will demonstrate that the results of these measurements are consistent to each other, giving the same critical exponent $\nu_{\|}$. Another important critical exponent is associated with the diverging lengthscale: $\xi \sim\left(a-a_{c}\right)^{-\nu_{\perp}}$. However, $\nu_{\perp}$ has not yet been measured in literature. In this paper, both static and dynamic correlation lengths $\xi$ are carefully studied for the first time and characterised quantitatively. In particular, they are both shown to diverge close to criticality with the same exponent $\nu_{\perp}$. A related critical exponent is the one of the susceptibility $\chi$ which quantifies the variance of the global fluctuations of the order parameter, and obyes $\chi \sim\left(a-a_{c}\right)^{-\gamma}$. Again, the exponent $\gamma$ has, to our knowledge, not been measured before, because it demands a proper (i.e. grand-canonical) estimate of the global fluctuations of the order parameter. The second aim of this work is to show that a large number of observables exist that can reveal directly the criticality of the model.

Thus both our model and the one introduced by Corté et al. [5] show a true dynamical phase transition from an irreversible to a reversible phase accompanied by diverging timescales, lengthscales and global fluctuations. By simultaneously measuring all critical exponents using various methods that appear to be internally consistent, we find these values to be consistent with both directed percolation (DP) and conserved directed percolation (CDP/Manna) universality class after appropriate finite size scaling. We emphasize that both universality classes share critical exponents that are very close to one another and are hard to distinguish numerically. Our results seem to favor the DP universality class, despite the presence of particle conservation, which is generally viewed as the key ingredient responsible for the emergence of the CDP universality class [2, 3]. We discuss this finding more extensively in the conclusion of the article.

Additionally, our simplified model also shows a true hyperuniformity at criticality, consistent to the conventional shear-based Corté model [9, 28, 29], which we show by a diverging hyperuniform lengthscale approaching criticality, as determined experimentally very recently [9]. We find, however, that this hyperuniform lengthscale is apparently unrelated to other critical lengthscales in the model. It is therefore unclear to us whether a deep connection exists between critical and hyperuniform fluctuations, although it does suggest a qualitative connection between fluctuations of the density and that of the order parameter.

Finally, we shall also point out a similarity between the dynamic criticality 


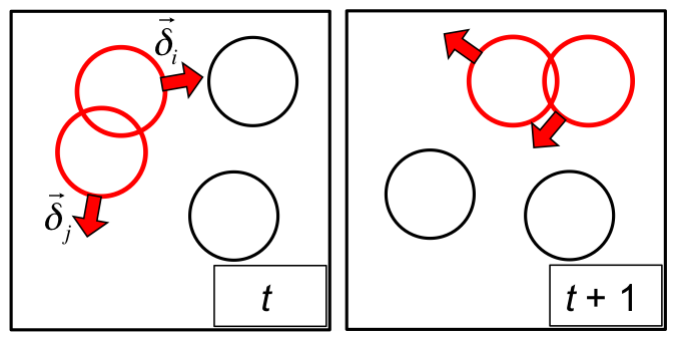

Figure 1. The model considered in our work: Particles overlapping at time $t$ (red) are labelled 'active' and are each given an independent random displacement $\vec{\delta}_{i}$. Particles with no overlap (black) are labelled 'passive' and are immobile, but may become active at a later time.

displayed in periodically driven systems near an irreversibility transition and dense liquids near a glass transition [30, 31]. We will show that both types systems exhibit a very heterogenous dynamics in space and time (which becomes critical at the reversible-irreversible transition). We find in particular that single-particle dynamics becomes intermittent and strongly non-Fickian, and that collective dynamics becomes spatially correlated over diverging lengthscales. The analogy between the two types of systems suggests that particle-based measurements and observables developed for glassy materials could prove useful in driven suspensions. These tools could in particular reveal whether the 'singularity-free' explanation based on the Lyapunov instability is experimentally relevant, as no special critical feature should appear in this case.

Our paper is organised as follows. In Sec. 2 we define the numerical model used in this study. The phase diagram and the order parameter critical exponent are analysed in Sec. 3. Static lengthscales and global fluctuations are measured in Sec. 4. We study single-particle and collective dynamics in Sec. 5 and conclude in Sec. 6 .

\section{Model}

The model considered in this paper is shown schematically in Fig. 1. We consider a 2-dimensional assembly of spherical particles of diameter $\sigma$ in a box of linear size $L$ with periodic boundary conditions. The system is initiated from a completely random configuration, where particle overlaps may exist. At each time step $t$, particles which overlap with at least one neighbour are labelled 'active' and we simultaneously move each active particle $i$ by an independent random displacement $\vec{\delta}_{i}$ :

$$
\vec{\delta}_{i}=\epsilon_{i} \hat{e}_{i}
$$

where $\hat{e}_{i}$ is a unit vector whose orientation is distributed randomly on a unit circle and the magnitude $\epsilon_{i}$ is a random number distributed uniformly on the interval $[0, \delta]$. Particles which do not overlap, on the other hand, are labelled 'passive' and remain immobile though they may become active and mobile later. The time $t$ is then incremented by one unit. The model has two control parameters: the area fraction $\phi=\pi N \sigma^{2} / 4 L^{2}$ and the maximal amplitude of the random kicks $\delta$. We use $\sigma$ as the 
unit length and we vary the area fraction by changing the number of particles $N$ while keeping the system size fixed at $L=280$ (unless mentioned otherwise). For this system size, we do not find significant finite size effect for the range of parameters that we use below. The value of the critical density obviously depends on the magnitude of the random kick $\delta$. We shall establish the phase diagram $\phi_{c}(\delta)$ in Sec. 3, but will conduct most of the our numerical studies of the critical behaviour of the system using $\delta=0.5$, for which $\phi_{c} \simeq 0.375$. We find that critical exponents are insensitive to the specific choice of $\delta$ and this value thus represents a compromise between too small values of $\delta$ which increase the computational time (because particles move very little), and much larger values of $\delta$, which become unphysical when $\delta \gg \sigma$.

Our model represents a simplified isotropic version of the periodically sheared suspension model considered in Ref. [5]. In [5], random kicks are given to all particles which collide during a shear deformation cycle. This original rule is in fact equivalent to giving a random kick to each particle having at least one neighbour in an anisotropic area near its centre [22]. In our model, we consider that this area is circular with $\sigma$ representing its diameter. This small simplification makes the determination of the critical properties of the model simpler because it prevents the development of locally anisotropic correlations [32], which could affect the numerical value of the measured exponents, but not the overall qualitative behaviour that we report. Our model may also represent an experimental situation in which a non-Brownian colloidal suspension is driven periodically by a periodic change of the particle diameters, leading to irreversible collisions. Such experiments could be realised using thermo-sensitive colloidal particles [8, 33, 34].

We perform each simulation run over $1.6 \times 10^{6}$ timesteps. Averages are taken over

$10^{6}$ timesteps in steady state. Close to criticality, we perform 4 independent simulation runs and if at least 1 run reaches an absorbing state before the maximum timestep is reached, the data for that density are discarded. The measurement itself is taken from a single run, using an extensive time averaging procedure.

\section{Order parameter and phase diagram}

We define the order parameter as the fraction of active particles at time $t$ :

$$
f_{a}(t)=\frac{N_{a}(t)}{N},
$$

where $N_{a}(t)$ is the total number of active/mobile particles (see Fig. 1).

Fig. 2(A) shows the evolution of the order parameter $f_{a}(t)$ (from an initially random configuration at $t=0)$. Below some critical density $\phi<\phi_{c}$, the number of active/mobile particles goes to zero as $t \rightarrow \infty$ (passive phase). The passive phase is also an absorbing state. This means once the number of active/mobile particles goes to zero, it is not possible to recover mobility. Physically, all overlaps (which are the source of mobility in our system) have been removed. On the other hand above the critical density $\phi>\phi_{c}$, the number of active/mobile particles remains finite and fluctuates around its mean $\left\langle f_{a}\right\rangle$ 

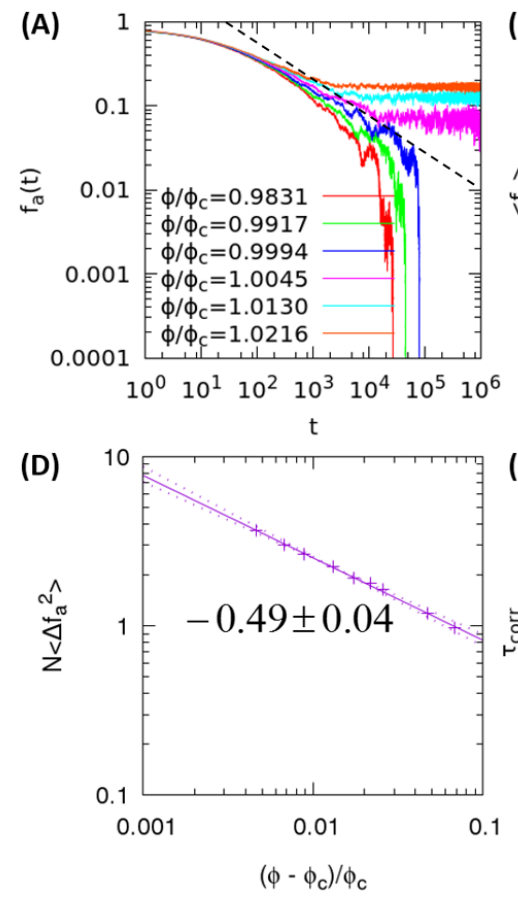
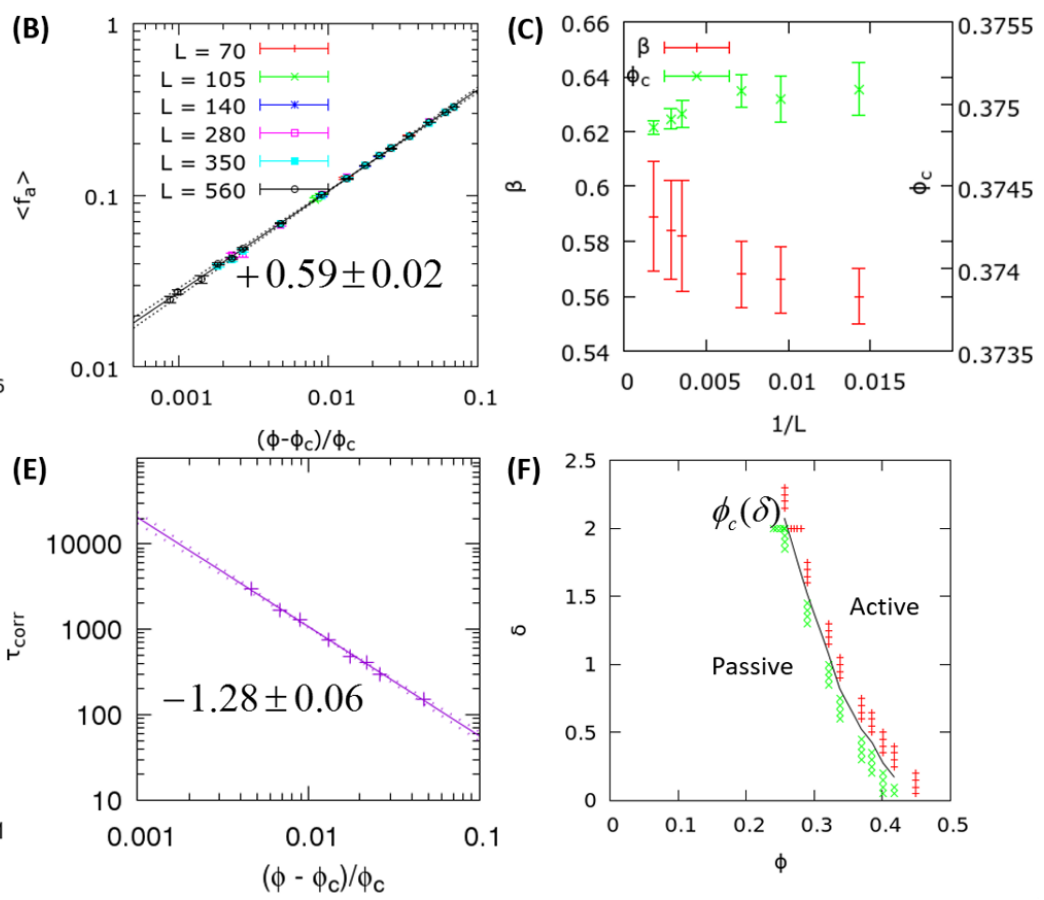

(F)

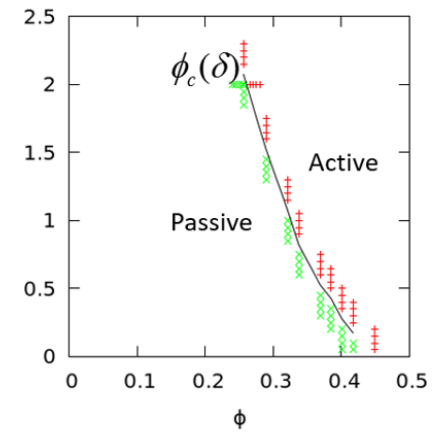

Figure 2. (A) Time dependence of the fraction of active particles from a random initial configuration. In the long-time limit, $f_{a}$ vanishes for $\phi<\phi_{c}$, fluctuates around a well-defined average above $\phi_{c}$. At criticality, a power law is observed, $f_{a}(t) \sim t^{-\alpha}$ with the exponent $\alpha \simeq 0.45$ (dashed line). (B) Algebraic vanishing of the average order parameter $\left\langle f_{a}\right\rangle$ with density for different system sizes $L,\left\langle f_{a}\right\rangle \sim\left(\phi-\phi_{c}\right)^{\beta}$, with $\beta \simeq 0.59 \pm 0.02$. The dashed lines indicate uncertainty in the critical exponent. (C) System size dependence of the critical exponent $\beta$ and critical density $\phi_{c}$. (D) Diverging fluctuations of the order parameter at steady state, $N\left\langle\Delta f_{a}^{2}\right\rangle \sim\left(\phi-\phi_{c}\right)^{-\gamma}$ with $\gamma \simeq 0.50$. (E) Diverging correlation time from steady state fluctuations of the order parameter, $\tau_{\text {corr }} \sim\left(\phi-\phi_{c}\right)^{-\nu_{\|}}$with $\nu_{\|} \simeq 1.27$. (F) Phase diagram on the parameter space $(\phi, \delta)$ separating the active phase (pluses) where the number of active particles remain finite at steady state and the absorbing phase (crosses) where the number of active particles drops to zero at steady state. The full line is a continuous line of equivalent second-order phase transitions.

in the steady state (active phase). At criticality $\phi=\phi_{c}$, the order parameter decays as power law: $f_{a}(t) \sim t^{-\alpha}$ with exponent $\alpha \simeq 0.45$, as indicated in Fig. 2(A). This value of $\alpha$ is close to the one reported in 2D directed percolation and conserved directed percolation universality classes [2].

The mean value $\left\langle f_{a}\right\rangle$ is simply defined to be the time average at steady state:

$$
\left\langle f_{a}\right\rangle=\frac{1}{T} \int_{t^{\prime \prime}}^{t^{\prime \prime}+T} d t^{\prime} f_{a}\left(t^{\prime}\right)
$$

where $t^{\prime \prime}$ is larger than the time it takes for the system reaches a steady state. Fig. 2(B) shows the average order parameter $\left\langle f_{a}\right\rangle$ as a function of packing fraction $\phi$ at different system sizes $L$. The $y$-errorbar in $\left\langle f_{a}\right\rangle$ is taken from square root of the fluctuation squared in $f_{a}(t)$ divided by the number of independent measurements (which is $T$ divided by the correlation time). The average order parameter is zero in the passive phase 
$\left(\phi<\phi_{c}\right)$ and positive in the active phase $\left(\phi>\phi_{c}\right)$. Furthermore from Fig. 2(B), we find that the average order parameter decreases continuously to zero at critical density $\phi_{c}$ with power law behaviour:

$$
\left\langle f_{a}\right\rangle \sim\left(\phi-\phi_{c}\right)^{\beta}, \quad \text { as } \phi \rightarrow \phi_{c}^{+} .
$$

The critical exponent $\beta$ is measured to be $\simeq 0.59 \pm 0.02$ and the critical density is $\phi_{c} \simeq 0.375 \pm 0.001$. Both are found by fitting a best straight fit line on the log-log scale (see Fig. 2(B)). The dashed lines in Fig. 2(B) indicate the \pm 0.02 uncertainty in the slopes or the critical exponent $\beta$. The system size dependence of $\beta$ and $\phi_{c}$ are plotted in Fig. 2(C). We found no significant finite size effects for these measurements, which depend only weakly on the system size. From the finite size scaling, we find that the critical exponent $\beta$ is consistent to both $2 \mathrm{D}$ directed percolation universality class $(\beta \simeq 0.5834)$ and conserved directed percolation or Manna universality class $(\beta \simeq 0.639)$ [2], although our data do appear to lie closer to the DP value. Although we cover a significant range of system sizes and correlation lengthscales (see below), we can of course not exclude the existence of a crossover at even larger lengthscale towards the CDP value, and even larger-scale simulations would be needed to see this. This would represent, we believe, a very significant numerical effort.

As we can see from Fig. 2(A), the fraction of active particles fluctuates around its mean value $\left\langle f_{a}\right\rangle$ in the active phase at steady state. This fluctuation becomes larger and diverges as we approach the critical density from above $\phi \rightarrow \phi_{c}^{+}$. We define the average fluctuation squared (or variance of the order parameter) to be:

$$
N\left\langle\Delta f_{a}^{2}\right\rangle=N\left\langle\left(f_{a}\left(t^{\prime}\right)-\left\langle f_{a}\right\rangle\right)^{2}\right\rangle,
$$

where the angle bracket indicates time averaging over $t^{\prime}$ at steady state, similar to Eq. (3). We plot the average fluctuation squared, $N\left\langle\Delta f_{a}^{2}\right\rangle$, as a function of $\phi$ in Fig. 2(D) which again exhibits a power law behaviour close to the critical point:

$$
N\left\langle\Delta f_{a}^{2}\right\rangle \sim\left(\phi-\phi_{c}\right)^{-\gamma}, \quad \text { as } \phi \rightarrow \phi_{c} .
$$

Similarly, the dashed lines indicate uncertainty in the critical exponent. However in this case, the measured critical exponent $(\gamma \simeq 0.49)$ appears quite different from that reported in both DP and CDP universality classes $(\gamma=0.30$ and 0.37 respectively [2]). This is because the global fluctuations of the order parameter in Fig. 2(D) are measured in the constrained ensemble where the total number of particles $N$ is fixed, which obviously affects the global fluctuations of any physical quantity [35]. In Sec. 4.2, we shall look at a more general fluctuation of the order parameter, computed in the grandcanonical ensemble. In that case, we will find that the critical exponent becomes very close to that of 2D directed percolation.

The time auto-correlation function of the order parameter is defined as:

$$
C(t)=\frac{\left\langle f_{a}\left(t^{\prime}+t\right) f_{a}\left(t^{\prime}\right)\right\rangle-\left\langle f_{a}\right\rangle\left\langle f_{a}\right\rangle}{\left\langle\Delta f_{a}^{2}\right\rangle},
$$

where the angle average indicates time averaging over $t^{\prime}$ at steady state as before. At $t=0$, the value of $C(t)$ is equal to 1 (maximum correlation) and, as $t$ increases, $C(t)$ 
decreases and eventually goes to zero in the limit $t \rightarrow \infty$ (completely decorrelated). The auto-correlation time of the order parameter $\tau_{\text {corr }}$ is defined by setting $C\left(\tau_{\text {corr }}\right)=e^{-1}$. The correlation time is shown to diverge near the critical point $\phi_{c}$ with a power law:

$$
\tau_{\text {corr }} \sim\left(\phi-\phi_{c}\right)^{-\nu_{\|}},
$$

where $\nu_{\|}=1.27$ (see Fig. $2(\mathrm{E})$ ). Again this value of $\nu_{\|}$is close to that of $2 \mathrm{D}$ directed percolation. Note that we report below the behaviour of other timescales and all these timescales were found to diverge with the same critical exponent $\nu_{\|}$, which we numerically find is close to $\nu_{\|} \simeq 1.26$. Such an agreement between critical exponents also holds for lengthscales (except for hyperuniform length), and similarly, we shall label the critical exponent associated with lengthscales with the unique notation $\nu_{\perp}$.

Fig. 2(F) shows the phase diagram on the parameter space $(\phi, \delta)$ showing the critical line $\phi_{c}(\delta)$ which separates the active phase from the passive phase. Each point on the line is a continuous/second order transition from passive to active phase. Furthermore, we have also found similar critical exponent $\beta \simeq 0.57 \pm 0.02$ for the order parameter $\left\langle f_{a}\right\rangle(\phi, \delta)$ at different points along the critical line going horizontally along $\phi$ or vertically along $\delta$. For the rest of this paper, we shall fix $\delta=0.5$ for which the critical density is $\phi_{c} \simeq 0.375$.

\section{Static properties}

\subsection{Structure factors and correlation lengthscales}

Fig. 3 shows the snapshot of the active phase far from critical density (left panel) and close to critical density (right panel), taken at steady state. The red circles indicate active/mobile particles and the grey circles indicate passive/immobile particles. Note that active particles can become passive after some time and vice versa. As we can see from the figure, active particles tend to cluster together and we shall define a 'static' lengthscale to be the typical size of these clusters, or equivalently, typical distance between two clusters. Comparing the two panels in Fig. 3, we observe a growing static lengthscale as we approach the critical density from above $\left(\phi \rightarrow \phi_{c}^{+}\right)$. In this section, we shall discuss how these static lengthscales can be measured. Note that in the experimental situation, the activity of the particle at time $t$ is determined over one cycle of oscillation. Thus the lengthscales discussed in this section are not purely static but instead determined by the dynamics of the particles over one cycle of oscillation. However for the purpose of the discussion, we shall call the lengthscales in this section as almost 'static' quantities. This is to distinguish such lengthscales from dynamic lengthscales in Sec. 5.2, which are measured over much larger time delays comprising many cycles.

Suppose we denote the number density of the active and passive particles to be $n_{a}(\mathbf{r}, t)$ and $n_{p}(\mathbf{r}, t)$ respectively. These can be written explicitly as:

$$
n_{a}(\mathbf{r}, t)=\sum_{i=1}^{N} \delta\left(\mathbf{r}-\mathbf{r}_{i}\right) f_{i}(t)=\sum_{i=1}^{N_{a}} \delta\left(\mathbf{r}-\mathbf{r}_{i}^{a}(t)\right),
$$



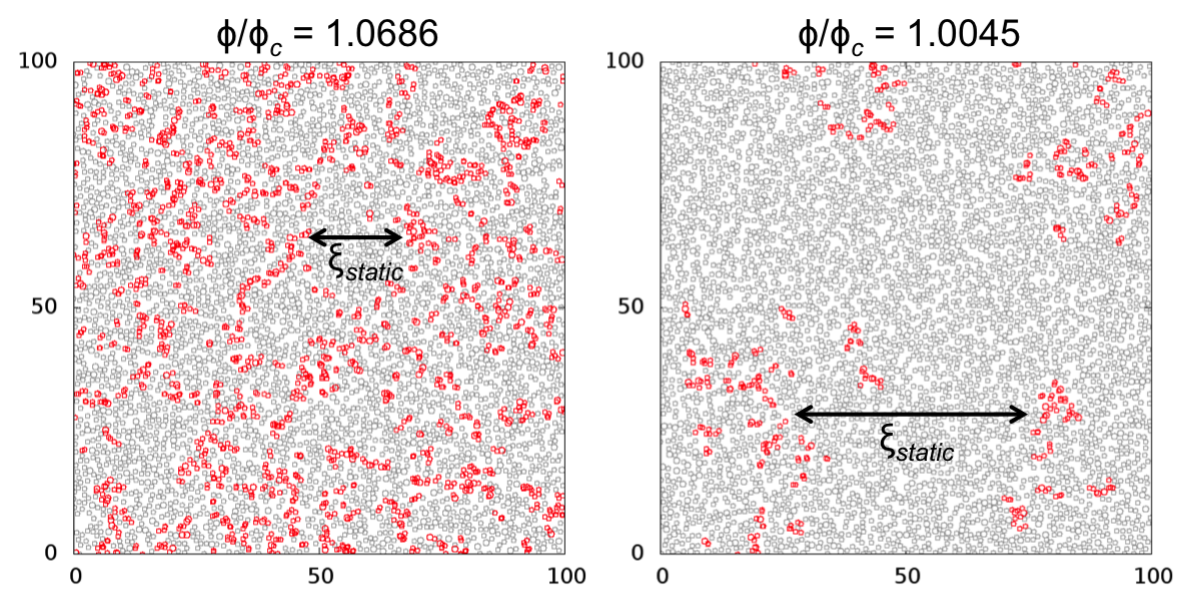

Figure 3. Snapshots of the active phase far above the critical density (left) and closer to the critical density (right). The red circles indicate particles which are active. As can be seen from the snapshots, we observe a growing static lengthscale as we approach the critical density of $\phi_{c} \simeq 0.375$.

$$
n_{p}(\mathbf{r}, t)=\sum_{i=1}^{N} \delta\left(\mathbf{r}-\mathbf{r}_{i}\right)\left(1-f_{i}(t)\right)=\sum_{i=1}^{N_{p}} \delta\left(\mathbf{r}-\mathbf{r}_{i}^{p}(t)\right),
$$

where $f_{i}(t)=1$ if particle $i$ is active at time $t$ and $f_{i}(t)=0$ if particle $i$ is passive at time $t ; \mathbf{r}_{i}^{a}(t)$ and $\mathbf{r}_{i}^{p}(t)$ are the positions (at time $t$ ) of the active and passive particles respectively. The average densities are simply:

$$
\left\langle n_{\alpha}(\mathbf{r}, t)\right\rangle=\frac{\left\langle N_{\alpha}\right\rangle}{V},
$$

where $\alpha=a, p$ refers to either active or passive particles. $V$ is the volume of the system and $\left\langle N_{a / p}\right\rangle$ is the averaged total number of active/passive particles respectively. The structure factors between the active particles $S_{a a}(q)$ and between the passive particles $S_{p p}(q)$ are defined to be [36]:

$$
S_{\alpha \alpha}(q)=\frac{1}{\left\langle N_{\alpha}\right\rangle}\left\langle\delta n_{\alpha}^{*}(q) \delta n_{\alpha}(q)\right\rangle
$$

where $\delta n_{\alpha}(q)$ is the Fourier transform of $\delta n_{\alpha}(\mathbf{r})=n_{\alpha}(\mathbf{r})-\left\langle n_{\alpha}\right\rangle$. By substituting Eq. (9) into (11), the structure factor can be computed explicitly as:

$$
S_{\alpha \alpha}(q)=\left\langle\frac{1}{N_{\alpha}} \sum_{i=1}^{N_{\alpha}} \sum_{j=1}^{N_{\alpha}} e^{i \mathbf{q} \cdot\left(\mathbf{r}_{i}^{\alpha}-\mathbf{r}_{j}^{\alpha}\right)}\right\rangle-\left\langle N_{a}\right\rangle \delta_{\mathbf{q}, 0} .
$$

The limit $q \rightarrow 0$ of the structure factors tells us about the fluctuations of the number of particles since:

$$
\frac{\left\langle\Delta N_{\alpha}^{2}\right\rangle}{\left\langle N_{\alpha}\right\rangle}=\frac{1}{\left\langle N_{\alpha}\right\rangle}\left\langle\delta n_{\alpha}^{*}(q \rightarrow 0) \delta n_{\alpha}(q \rightarrow 0)\right\rangle=S_{\alpha \alpha}(q \rightarrow 0) .
$$

Finally we can define the mixed structure factor between the active and passive particles to be:

$$
S_{p a}(q)=\frac{1}{\sqrt{\left\langle N_{p}\right\rangle\left\langle N_{a}\right\rangle}} \operatorname{Re}\left\langle\delta n_{p}^{*}(q) \delta n_{a}(q)\right\rangle
$$



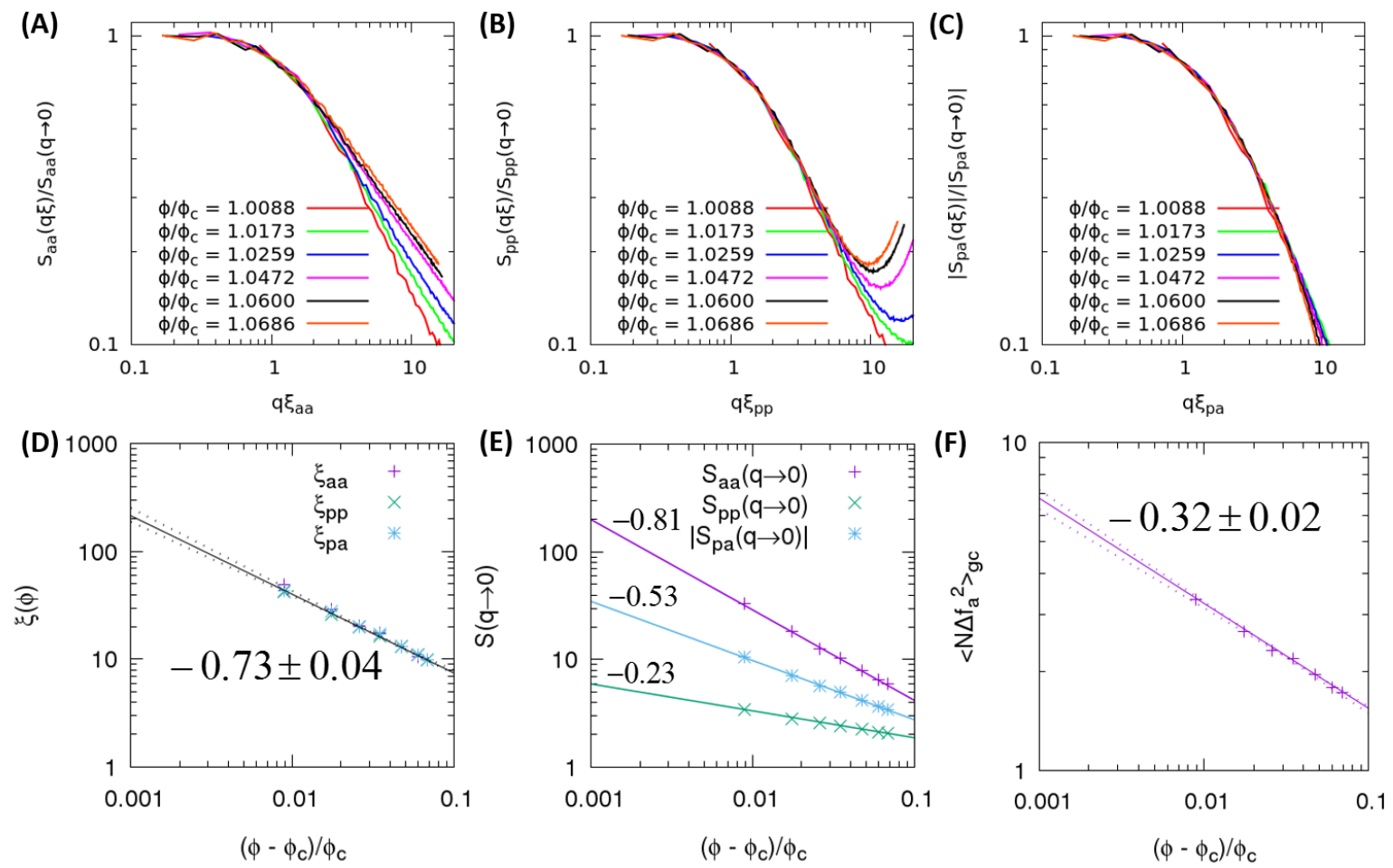

Figure 4. (A,B,C) Scaling analysis of partial structure factors between activeactive particles $S_{a a}(q)$, passive-passive particles $S_{p p}(q)$ and passive-active particles $S_{p a}(q)$ using Eq. (16). This data collapse allows for the determination of a correlation lengthscale $\xi_{\alpha \beta}(\phi)$. (D) The measured lengthscales $\xi_{\alpha \beta}(\phi)$ diverge as $\xi \sim\left(\phi-\phi_{c}\right)^{-\nu_{\perp}}$, with the same critical exponent $\nu_{\perp} \simeq 0.73$, close to that of $2 \mathrm{D}$ directed percolation. The dashed lines indicate uncertainty in the critical exponent. (E) Global number densitiy fluctuations, $S_{\alpha \beta}(q \rightarrow 0)$, also diverge with power laws as we approach $\phi_{c}$, with three distinct critical exponents. (F) The appropriate combination of $S_{a a}(q \rightarrow 0)$, $S_{p p}(q \rightarrow 0)$ and $S_{p a}(q \rightarrow 0)$ in Eq. (25) provides the order parameter fluctuations, $\left\langle N \Delta f_{a}^{2}\right\rangle_{g c}$, which diverges as $\chi=\left\langle N \Delta f_{a}^{2}\right\rangle_{g c} \sim\left(\phi-\phi_{c}\right)^{-\gamma}$ with $\gamma \simeq 0.31$, in good agreement with DP universality class.

$$
=\frac{1}{\sqrt{\left\langle N_{p}\right\rangle\left\langle N_{a}\right\rangle}} \operatorname{Re}\left\langle\sum_{i=1}^{N_{p}} \sum_{j=1}^{N_{a}} e^{i \mathbf{q} \cdot\left(\mathbf{r}_{i}^{p}-\mathbf{r}_{j}^{a}\right)}\right\rangle-\sqrt{\left\langle N_{p}\right\rangle\left\langle N_{a}\right\rangle} \delta_{\mathbf{q}, 0} .
$$

The limit $q \rightarrow 0$ of $S_{p a}(q)$ gives us the cross-correlation of the number fluctuations:

$$
S_{p a}(q \rightarrow 0)=\frac{\left\langle\Delta N_{a} \Delta N_{p}\right\rangle}{\sqrt{\left\langle N_{a}\right\rangle\left\langle N_{p}\right\rangle}} .
$$

In general the fluctuations squared of the number density $S_{\alpha \beta}(q \rightarrow 0)$ depend on the packing fraction $\phi$ which diverges as $\phi \rightarrow \phi_{c}^{+}$. In fact in critical phenomena, the structure factors can be rescaled by a lengthscale $\xi(\phi)$ such that all structure factors at different packing fractions $\phi$ can be collapsed into a single universal function $F(x)$ which does not depend on $\phi$ :

$$
\frac{S_{\alpha \beta}(q \xi(\phi))}{S_{\alpha \beta}(q \rightarrow 0, \phi)}=F_{\alpha \beta}(q \xi(\phi))
$$


which should be true as long as $q$ is small compared to $2 \pi / \xi$. The structure factors of active-active particles $S_{a a}(q)$, passive-passive particles $S_{p p}(q)$ and passive-active particles $S_{p a}(q)$ for different values of $\phi>\phi_{c}$ are plotted in Figs. 4(A,B,C). As can be seen from the figure, all structure factors from different densities can indeed be collapsed into a single curve, from which, a lengthscale $\xi_{\alpha \beta}(\phi)$ can be extracted and is shown to diverge with a power law:

$$
\xi \sim\left(\phi-\phi_{c}\right)^{-\nu_{\perp}}, \quad \text { as } \phi \rightarrow \phi_{c}^{+},
$$

(see Fig. 4(D)). We assume all lengthscales are equivalent: $\xi_{a a} \propto \xi_{p p} \propto \xi_{p a}$ and we found the average critical exponent for the static lengthscale to be $\nu_{\perp} \simeq 0.73$, consistent to that of 2D directed percolation (see Fig. 4(D)).

Finally, the number density fluctuations of active, passive, or mixed particles can be found from the limit $S_{\alpha \beta}(q \rightarrow 0)$ (see Fig. $4(\mathrm{E})$ ). These number fluctuations are also shown to be power law divergent as we approach the critical density with three distinct critical exponents as indicated in the figure. The ratio of the lengthscales $\xi_{\alpha \beta}$ to number fluctuations $S_{\alpha \beta}(q \rightarrow 0)$ is related to another critical exponent which we shall call $\theta_{\alpha \beta}$. More precisely, we define $\theta_{\alpha \beta}$ to be:

$$
S_{\alpha \beta}(q \rightarrow 0)=\xi_{\alpha \beta}^{2-\theta_{\alpha \beta}} .
$$

For active-active this value is $\theta_{a a}=0.97$, for passive-passive $\theta_{p p}=1.67$ and finally for mixed passive-active $\theta_{p a}=1.28$.

\subsection{Grand canonical fluctuations in the order parameter}

In Sec. 3, we defined the order parameter to be the fraction of active particles at time $t$ :

$$
f_{a}(t)=\frac{N_{a}(t)}{N} \quad(\text { canonical })
$$

where the total number of particles in our system is fixed: $N=$ constant (i.e. canonical ensemble). Thus, the only source of fluctuations in the order parameter comes from the total number of active particles $N_{a}(t)$. Subsequently, we have also found that the canonical fluctuations diverge near the critical density as power law: $N\left\langle\Delta f_{a}^{2}\right\rangle \propto\left(\phi-\phi_{c}\right)^{-\gamma}$ with a critical exponent $\gamma=0.50$.

On the other hand, we could have also defined the order parameter in the grandcanonical ensemble where the total number of particles is not constant, but fluctuates around its mean value $\langle N\rangle$ :

$$
f_{a}(t)=\frac{N_{a}(t)}{N(t)} \quad(\text { grand }- \text { canonical }) .
$$

The mean value of the order parameter $\left\langle f_{a}\right\rangle$ is the same whether it is taken in the canonical or grand-canonical ensemble: $\left\langle f_{a}\right\rangle=\left\langle f_{a}\right\rangle_{g c}$. (The subscript $\langle\cdot\rangle_{g c}$ in this section indicates time averaging in the grand canonical ensemble.) However the averaged fluctuations squared of the order parameter are different: $\left\langle\Delta f_{a}^{2}\right\rangle \neq\left\langle\Delta f_{a}^{2}\right\rangle_{g c}$ since fluctuations in $N(t)$ will now contribute to fluctuations in $f_{a}(t)$. 
We may obtain the grand canonical fluctuation squared of the order parameter from the partial structure factors indirectly as follow. First we write the order parameter density to be:

$$
f_{a}(\mathbf{r}, t)=\frac{n_{a}(\mathbf{r}, t)}{n_{a}(\mathbf{r}, t)+n_{p}(\mathbf{r}, t)} .
$$

Thus the average fluctuation over the whole system volume is:

$$
\begin{aligned}
\Delta f_{a}(t) & =\frac{1}{V} \int_{V} d V \delta f_{a}(\mathbf{r}, t) \\
& =\frac{1}{V} \delta f_{a}(q=0, t),
\end{aligned}
$$

where $\delta f_{a}=f_{a}-\left\langle f_{a}\right\rangle$. Therefore the fluctuations squared of the order parameter in the grand-canonical scheme is given by:

$$
\left\langle N \Delta f_{a}^{2}\right\rangle_{g c}=\frac{\langle N\rangle}{V^{2}}\left\langle\delta f_{a}^{*}(q \rightarrow 0) \delta f_{a}(q \rightarrow 0)\right\rangle
$$

Writing $\delta f_{a}$ in terms of $\delta n_{a}=n_{a}-\left\langle n_{a}\right\rangle$ and $\delta n_{p}=n_{p}-\left\langle n_{p}\right\rangle$, we have:

$$
\delta f_{a}=\frac{V}{\langle N\rangle}\left[\left(1-\left\langle f_{a}\right\rangle\right) \delta n_{a}-\left\langle f_{a}\right\rangle \delta n_{p}\right]
$$

and substituting Eq. (24) into (23), the grand canonical fluctuations squared of the order parameter can be expressed in terms of the partial structure factors as follows [35, 36]:

$$
\begin{aligned}
\left\langle N \Delta f_{a}^{2}\right\rangle_{g c}= & \left(1-\left\langle f_{a}\right\rangle\right)^{2}\left\langle f_{a}\right\rangle S_{a a}(q \rightarrow 0)+\left\langle f_{a}\right\rangle^{2}\left(1-\left\langle f_{a}\right\rangle\right) S_{p p}(q \rightarrow 0) \\
& -2\left[\left\langle f_{a}\right\rangle\left(1-\left\langle f_{a}\right\rangle\right)\right]^{3 / 2} S_{p a}(q \rightarrow 0) .
\end{aligned}
$$

Physically, this formula estimates the fluctuations of the order parameter that would be measured if we were observing a subsystem of our model immersed in a much larger system size [35], as would be done in an experiment, for instance. We emphasize that 'grand-canonical' does not mean that the number of particles is no longer conserved, simply that the fluctuations inside a subsystem only arise via exchange with the reservoir. Therefore, Eq. 25 provides the correct estimate of the global fluctuations of the order parameter for our model for which the microscopic dynamics conserves particles. Thus, from the limit $q \rightarrow 0$ of the partial structure factors $S_{\alpha \beta}$ in Fig. 4(E), we obtain the grand canonical fluctuation squared of the order parameter $\left\langle N \Delta f_{a}^{2}\right\rangle_{g c}$, shown in Fig. 4(F). We now find that the grand-canonical fluctuations squared of the order parameter diverges as $\left\langle N \Delta f_{a}^{2}\right\rangle_{g c} \sim\left(\phi-\phi_{c}\right)^{-\gamma_{g c}}$ with the critical exponent $\gamma_{g c} \simeq 0.32$. This is in good agreement to that of $2 \mathrm{D}$ directed percolation, and thus resolves the apparent discrepancy of the canonical critical exponent $\gamma \simeq 0.50 \mathrm{in} \mathrm{Sec.} 3$.

\subsection{Hyperuniform scaling}

Consider a grand-canonical system with volume $V=L^{d}$, where $d$ is the dimension of the system $(d=2$ in our case). The system can exhange particles with the reservoir and consequently the total number of particles fluctuates around its mean $\langle N\rangle$. For 
a Poisson process, the variance of the total number of particles scales as the system volume:

$$
\left\langle\Delta N^{2}\right\rangle \sim L^{d}
$$

whereas for a hyperuniform system, the fluctuation is suppressed:

$$
\left\langle\Delta N^{2}\right\rangle \sim L^{d-\lambda}
$$

where $\lambda \in[0,1]$ is the hyperuniform exponent [37]. The exponent value of $\lambda=1$ has been reported in hard sphere systems close to the jamming transition [38, 39]. However, it has also been suggested recently in [40, 41] that true hyperuniformity might not survive in the limit of large system size at the jamming transition. On the other hand in periodically sheared suspension [5], true hyperuniformity has been reported numerically by Hexner and Levine [28, and experimentally by Weijs et al. [9] with hyperuniform exponent $\lambda \approx 0.45$. Here we report that the value of $\lambda=0.45$ is only a crossover value found at intermediate range of $q$-vector and we report an even stronger hyperuniformity with exponent $\lambda=1$ [21].

The total structure factor is defined as:

$$
S(q)=\left\langle\frac{1}{N} \sum_{i=1}^{N} \sum_{j=1}^{N} e^{i \mathbf{q} \cdot\left(\mathbf{r}_{i}-\mathbf{r}_{j}\right)}\right\rangle-\langle N\rangle \delta_{\mathbf{q}, 0} .
$$

A signature of hyperuniform scaling is the behaviour of the total structure factor at low $q$ :

$$
S(q) \propto q^{\lambda}, \quad q \rightarrow 0 .
$$

since the limit $q \rightarrow 0$ of the structure factor $S(q)$ is related to the number density fluctuation squared: $S(q \rightarrow 0)=\left\langle\Delta N^{2}\right\rangle /\langle N\rangle$.

We show in Figs. $5(\mathrm{~A}, \mathrm{~B})$ the total structure factor $S(q)$ as a function of $q$ for different densities above and below the critical density $\phi_{c}$. Approaching criticality from above $\phi \rightarrow \phi_{c}^{+}$(Fig. 5(A)), we observe a clear signature of hyperuniform scaling: $S(q) \propto q^{\lambda}$ with hyperuniform exponent $\lambda=0.45$ crossing over to $\lambda=1$ at lower values of $q$. Note that the intermediate value of $\lambda=0.45$ is the one that has been reported in Ref. [28]. Similarly approaching criticality from below $\phi \rightarrow \phi_{c}^{-}$(Fig. 5(B)), we also observe a clear hyperuniform scaling with exponent $\lambda=0.45$ crossing over to $\lambda=1$. Note that for $\phi<\phi_{c}$, we perform several simulations from different initial random configurations and wait until each run goes into a different absorbing state. The static structure factor is then ensemble-averaged over different runs. For $\phi>\phi_{c}$, long time average at steady state is sufficient since the system is dynamically exploring its available phase space.

Finally, following Ref. [9], we may define a hyperuniform length scale $\ell_{H}(\phi)$ to be the largest system size below which hyperuniform scaling holds for a given density $\phi$. $\ell_{H}$ can be obtained from the smallest value of $q$-vector at which the structure factor $S(q)$ starts to deviate from the hyperuniform scaling: $S(q) \sim q^{\lambda}$. We find that the hyperuniform length scale tends to diverge as we approach criticality both from below and above $\phi \rightarrow \phi_{c}^{ \pm}$(see Fig. 5(C)). This implies that true hyperuniformity might survive 

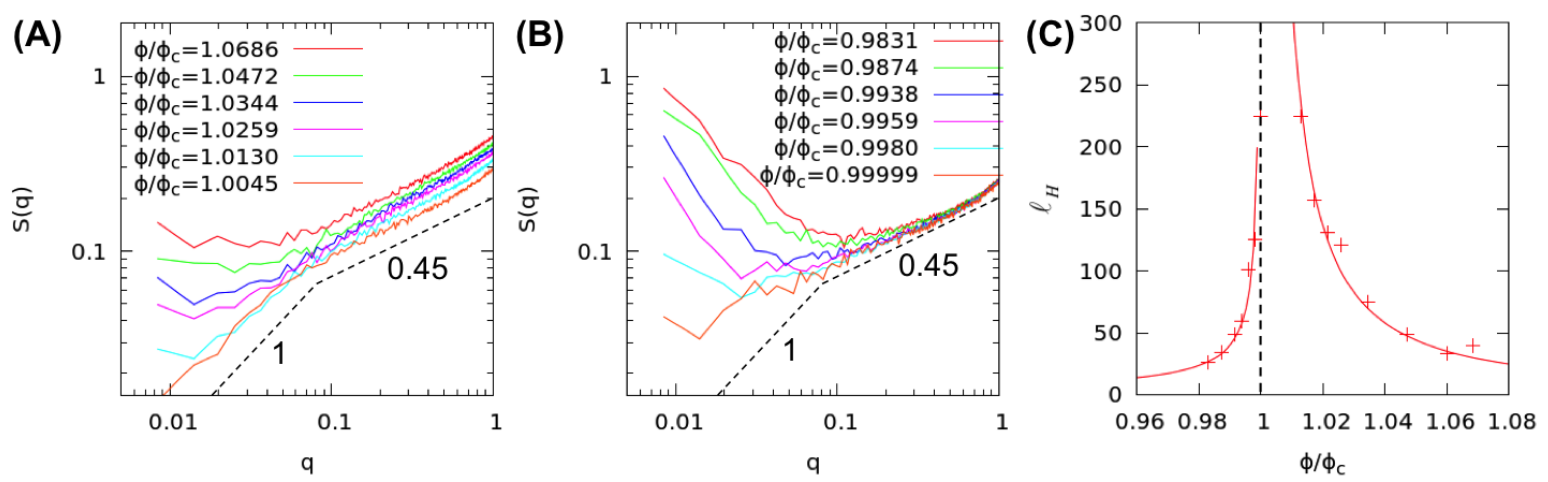

Figure 5. Hyperuniform scaling close to criticality $\phi_{c}$. (The system size used in this case is $L=560$.) (A) Total structure factor $S(q)$ for different densities as we approach the critical density from above $\phi \rightarrow \phi_{c}^{+}$. Close to criticality, we detect a clear signature of hyperuniformity $S(q) \propto q^{\lambda}$ with hyperuniform exponent $\lambda=0.45$ crossing over to $\lambda=1$ at lower $q$. (B) Total structure factor as we approach the critical density from below $\phi \rightarrow \phi_{c}^{-}$. Again we observe hyperuniform scaling with exponent 0.45 crossing over to 1 . (C) Hyperuniform length scale $\ell_{H}$ defined as the largest system size below which hyperuniform scaling holds for a given density $\phi$. It appears to diverge asymmetrically from both sides of $\phi_{c}$, suggesting that a true hyperuniform structure exists precisely at criticality.

in the large system size limit at $\phi_{c}$. By fitting power law: $\ell_{H} \sim\left|\phi-\phi_{c}\right|^{-\nu_{H}}$ we find the critical exponent for $\ell_{H}$ to be $\nu_{H} \simeq 0.76$ for $\phi \rightarrow \phi_{c}^{-}$and $\nu_{H} \simeq 1.23$ for $\phi \rightarrow \phi_{c}^{+}$. Note that the critical exponent of the hyperuniform lengthscale $\nu_{H}$ is different to those of static and dynamic lengthscales $\nu_{\perp}$, and also appears to be different on both sides of the transition. One reason explaining this asymmetry lies in the fact that the final structure of absorbing states below $\phi_{c}$ crucially depends on the initial configurations, whereas above $\phi_{c}$ the phase space is explored dynamically in a nonequilibrium steady state. A recent numerical work has shown that the configurations explored by the dynamics below $\phi_{c}$ differ from an equilibrium sampling of the available phase space [29]. (Note that the work of Ref. [29] would have been technically much easier-but conceptually equivalent-using our isotropic version of the model by Corté et al., since it would only involve canonical hard sphere simulations.)

\subsection{Pair distribution functions}

The pair distribution function between active-active or passive-passive particles is defined to be [36]:

$$
g_{\alpha \alpha}(r)=\frac{1}{\left\langle n_{\alpha}\right\rangle}\left\langle\sum_{i=1}^{N_{\alpha}} \sum_{j \neq i} \delta\left(\mathbf{r}-\left(\mathbf{r}_{i}-\mathbf{r}_{j}\right)\right)\right\rangle,
$$

where $\alpha=a, p$ refers to active or passive particles respectively. Physically, $\left\langle n_{\alpha}\right\rangle g_{\alpha \alpha}(r)$ tells us the average density of $\alpha$ particles at a distance $r$ from a given particle of the same species $\alpha$. Note that for isotropic and homogenous systems, $g_{\alpha \alpha}(r)$ only depends on the radial distance $r$. Furthermore we also have the asymptotic limit: $g_{\alpha \alpha}(r \rightarrow \infty)=1$, 
since the particles become uncorrelated at large distance. It can be easily shown that $g_{\alpha \alpha}(r)$ is related to the inverse Fourier transform of the structure factors $S_{\alpha \alpha}(q)$ defined in Eq. (12):

$$
h_{\alpha \alpha}(r)=g_{\alpha \alpha}(r)-1=\frac{1}{\left\langle n_{\alpha}\right\rangle} \frac{1}{(2 \pi)^{2}} \int d \mathbf{q} e^{-i \mathbf{q} \cdot \mathbf{r}}\left\{S_{\alpha \alpha}(q)-1\right\},
$$

where we have defined, for convenience, $h_{\alpha \alpha}(r)=g_{\alpha \alpha}(r)-1$. Similarly, the mixed pair distribution function between active and passive particles is defined to be:

$$
g_{p a}(r)=\frac{1}{\sqrt{\left\langle n_{p}\right\rangle\left\langle n_{a}\right\rangle}}\left\langle\sum_{i=1}^{N_{a}} \sum_{j=1}^{N_{p}} \delta\left(\mathbf{r}-\left(\mathbf{r}_{i}^{a}-\mathbf{r}_{j}^{p}\right)\right)\right\rangle .
$$

Physically, $\left\langle n_{p}\right\rangle g_{p a}(r)$ gives us the average density of passive particles at a distance $r$ from a given active particle (and vice versa since $g_{p a}(r)=g_{a p}(r)$ ). Again, the mixed pair distribution function is related to the inverse Fourier transform of the mixed structure factor $S_{p a}(q)$ defined in Eq. (14):

$$
h_{p a}(r)=g_{p a}(r)-1=\frac{1}{\sqrt{\left\langle n_{p}\right\rangle\left\langle n_{a}\right\rangle}} \frac{1}{(2 \pi)^{2}} \int d \mathbf{q} e^{-i \mathbf{q} \cdot \mathbf{r}} S_{p a}(q) .
$$

For active-active particles, the pair distribution function $g_{a a}(r)$ is plotted in Fig. 6(A). As can be seen from the figure, $g_{a a}(r \rightarrow \infty)=1$ as expected. However the value of $g_{a a}(r \simeq 1)$ diverges as $\phi \rightarrow \phi_{c}^{+}$. This suggests clustering of active particles as we approach critical density. Furthermore we have verified that $g_{a a}(r \simeq 1)$ scales as $1 /\left\langle n_{a}\right\rangle$, so that the density of the active particles inside the cluster $\left(=\left\langle n_{a}\right\rangle g_{a a}(r=1)\right)$ is independent of the density of the whole system. This is as we expect because the active particles inside the cluster behave like an ideal gas, and consequently, the density of the cluster only depends on the size of the random kick $\delta$. Similarly from the definition of passive-active pair distribution function, Eq. (32), we expect $g_{p a}(r \simeq 1)$ to scale as $1 / \sqrt{\left\langle n_{a}\right\rangle\left\langle n_{p}\right\rangle}$. The pair distribution function for the active-active, passive-active and passive-passive are plotted in Figs. 6(A,B,C) respectively. The pair distribution function for the passive-passive case (Fig. 6(C)) resembles somewhat that of hard spheres system. This is because all passive particles do not overlap with each other, thus behaving qualitatively similarly to a hard sphere fluid, with quantitative differences [29].

From the snapshots in Fig. 3, we see a growing static correlation length as we approach criticality. This is reflected both in the structure factors (as we have seen from previous subsection) and in the pair distribution function. In the case of the pair distribution function, we expect $g_{\alpha \beta}(r)$ to decay as $g_{\alpha \beta}(r) \sim e^{-r / \xi_{\alpha \beta}}$ for large $r$ where $\xi_{\alpha \beta}$ is the static correlation length. For instance, $\xi_{a a}$ gives us the typical size of the active clusters and $\xi_{p a}$ gives us the typical distance between the clusters. Subsequently close to criticality, we may assume the following asymptotic form for the pair distribution function:

$$
\frac{h_{\alpha \beta}(r)}{h_{\alpha \beta}\left(r=1^{+}, \phi\right)} \simeq \frac{1}{r^{\theta_{\alpha \beta}^{\prime}}} e^{-\frac{r}{\xi_{\alpha \beta}(\phi)}}, \quad \text { for } r \gg \xi
$$



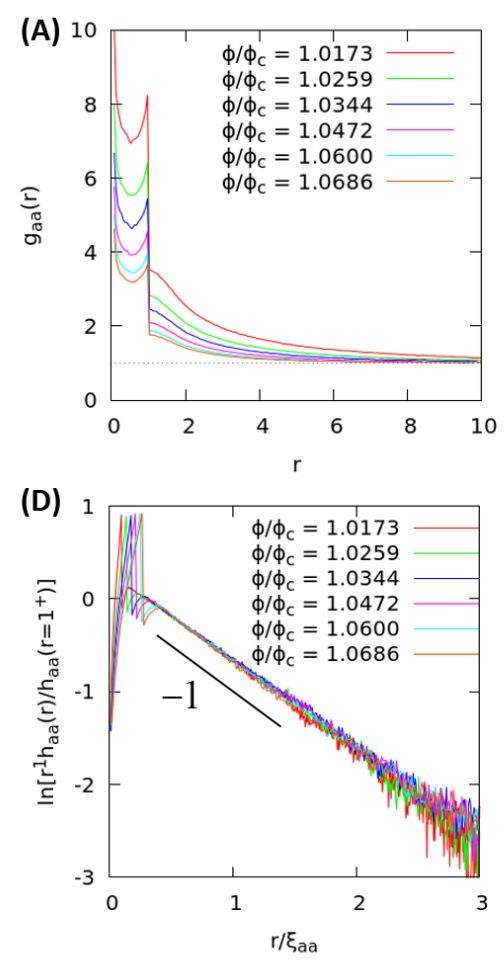
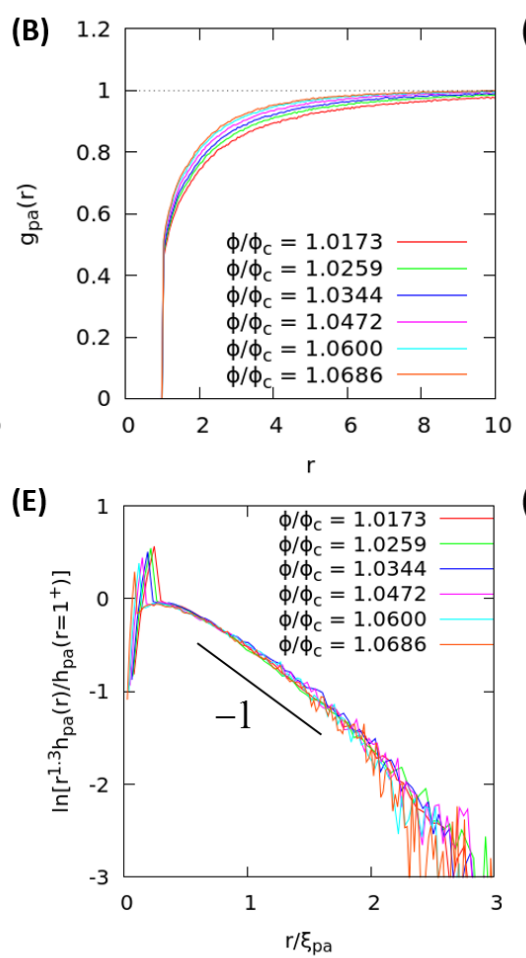
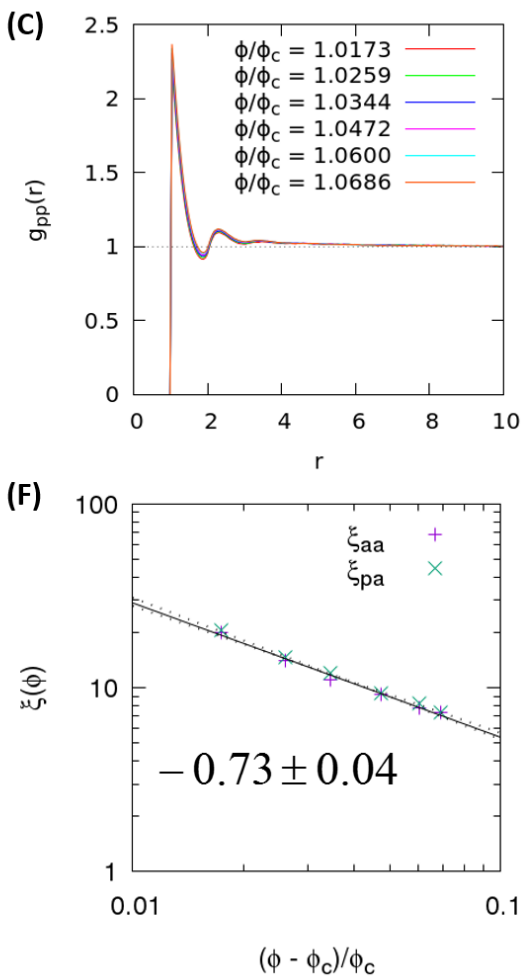

Figure 6. (A,B,C) Pair distribution functions for active-active particles $g_{a a}(r)$, passive-active particles $g_{p a}(r)$ and passive-passive particles $g_{p p}(r)$. (D,E) The pair distribution functions can be rescaled by the appropriate lengthscales $\xi_{\alpha \beta}(\phi)$ (see text for the scaling form used to fit the functions). (F) The extracted lengthscales for active-active particles (red plus) and passive-active particles (green cross) diverge as $\xi \sim(\phi-\phi)^{-\nu_{\perp}}$ with $\nu_{\perp} \simeq 0.73$.

where we know from above, $h_{\alpha \beta}\left(r=1^{+}\right)$scales as $1 /\left\langle n_{a}\right\rangle$ for the active-active case and $1 / \sqrt{\left\langle n_{a}\right\rangle\left\langle n_{p}\right\rangle}$ for the passive-active case. The exponent $\theta^{\prime}$ in the equation above can be derived from the structure factors as follows. First we recognize that the asymptotic limit $r \gg \xi$ is equivalent to the limit $q \ll \frac{2 \pi}{\xi}$ in the Fourier space. For example, the active-active pair distribution function $h_{a a}(r)$ can be obtained from the Fourier transform of the structure factor as follows:

$$
\begin{aligned}
S_{a a}(q) & =1+\left\langle n_{a}\right\rangle \int d^{2} r e^{i \mathbf{q} \cdot \mathbf{r}} h_{a a}(r) \\
& \simeq 1+A \int d^{2} r e^{i \mathbf{q} \cdot \mathbf{r}} \frac{1}{r^{\theta_{a a}^{\prime}}} e^{-\frac{r}{\xi_{a a}(\phi)}} \quad \text { for } q \ll \frac{2 \pi}{\xi_{a a}},
\end{aligned}
$$

where $A=\left\langle n_{a}\right\rangle h_{a a}\left(r=1^{+}\right)$is some constant which does not depend on $\phi$ (since $h_{a a}\left(r=1^{+}\right)$scales as $\left.1 /\left\langle n_{a}\right\rangle\right)$. Finally by change of variable: $u=r / \xi_{a a}$, we have for small $q \ll \frac{2 \pi}{\xi_{a a}}$ :

$$
S_{a a}(q) \simeq 1+\xi_{a a}(\phi)^{2-\theta_{a a}^{\prime}} \underbrace{A \int d^{2} u e^{i \mathbf{q} \xi_{a a}(\phi) \cdot \mathbf{u}} \frac{1}{u^{\theta_{a a}^{\prime}}} e^{-u}}_{J\left(q \xi_{a a}(\phi)\right)}, \quad \text { for } q \ll \frac{2 \pi}{\xi_{a a}} .
$$


where $J(x)$ is a universal function which does not depend on $\phi$ (compare to Eq. (16)). Therefore in the limit $q \rightarrow 0$, we have

$$
S_{a a}(q \rightarrow 0) \simeq 1+\xi_{a a}(\phi)^{2-\theta_{a a}^{\prime}} J(0),
$$

and comparing this equation to Eq. (18), we deduce that $\theta_{a a}^{\prime}=\theta_{a a}$ (similarly for the passive-active case $\left.\theta_{p a}^{\prime}\right)$. Imposing the values of $\theta_{a a}=1$ and $\theta_{p a}=1.3$ obtained from the structure factors, we may collapse the pair distribution functions into the scaling form in Eq. (34), shown below, suggesting that our measurements are at least internally consistent. These measurements cannot provide a very accurate estimate of the exponent $\theta_{a} a$ to be used in the distinction between DP and CDP universality classes.

In Fig. 6(D), we plot $\ln \left[r \frac{h_{a a}(r)}{h_{a a}\left(r=1^{+}, \phi\right)}\right]$ as a function of $\frac{r}{\xi_{a a}(\phi)}$. By choosing the appropriate values for $\xi_{a a}(\phi)$, we can collapse all pair distribution functions for different $\phi$ 's into a single straight line with slope -1 . From this universal scaling, we extract a lengthscale $\xi_{a a}(\phi)$ which has power law divergence close to $\phi_{c}$ (see Fig. 6(F)). Similarly for the mixed passive-active case, we plot $\ln \left[r^{1.3} \frac{h_{p a}(r)}{h_{p a}\left(r=1^{+}, \phi\right)}\right]$ as a function of $\frac{r}{\xi_{p a}(\phi)}$ (see Fig. 6(E)). From the data collapse, we extract the lengthscale $\xi_{p a}(\phi)$ which is plotted in Fig. 6(F). Assuming both lengthscales are equivalent, we find the critical exponent for the lengthscale to be: $\nu_{\perp} \simeq 0.73$ (see Fig. 6(F)). Note that this value of the critical exponent is indeed consistent to the one obtained from the structure factors in Sec. 4.1. Finally it should be mentioned that, for the passive-passive case, the values of the pair distribution function for different $\phi$ 's are too close to each other and no good statistics could be obtained to extract the lengthscale.

\section{Dynamic properties and heterogeneities}

\subsection{Single particle dynamic heterogeneities}

The dynamics of the system at the particle scale can be characterised by two timescales. 1) The timescale for an active particle to become passive $\left(\tau_{p}\right)$ and 2$)$ the timescale for a passive particle to become active $\left(\tau_{a}\right)$. The passivation timescale $\tau_{p}$ is typically much shorter and does not depend on the packing fraction. In fact, the passivation timescale only depends on the size of the random displacement $\delta$. On the other hand the activation timescale $\tau_{a}$ becomes longer and diverges as we approach the critical packing density from above $\left(\phi \rightarrow \phi_{c}^{+}\right)$.

Fig. 7 shows the typical trajectory of a single particle (in the $x$-direction). From the trajectory, we can see that the particle remains stationary (passive) for some time before moving (active) and so on. The activation timescale $\tau_{a}$ is thus the typical timescale for a single particle to remain stationary/passive. Similarly, the passivation timescale $\tau_{p}$ is the typical timescale for the particle to remain mobile/active (see left panel in Fig. 7). (This is analogous to the trajectory of the particles in glassy systems where the particle is caged by their neighbours and there exists an activation timescale for the particle to escape the cage [42].) Over large distances and times as compared to $\tau_{a}$, the trajectory of the particle eventually becomes indistinguishable from that of a random walker (see 

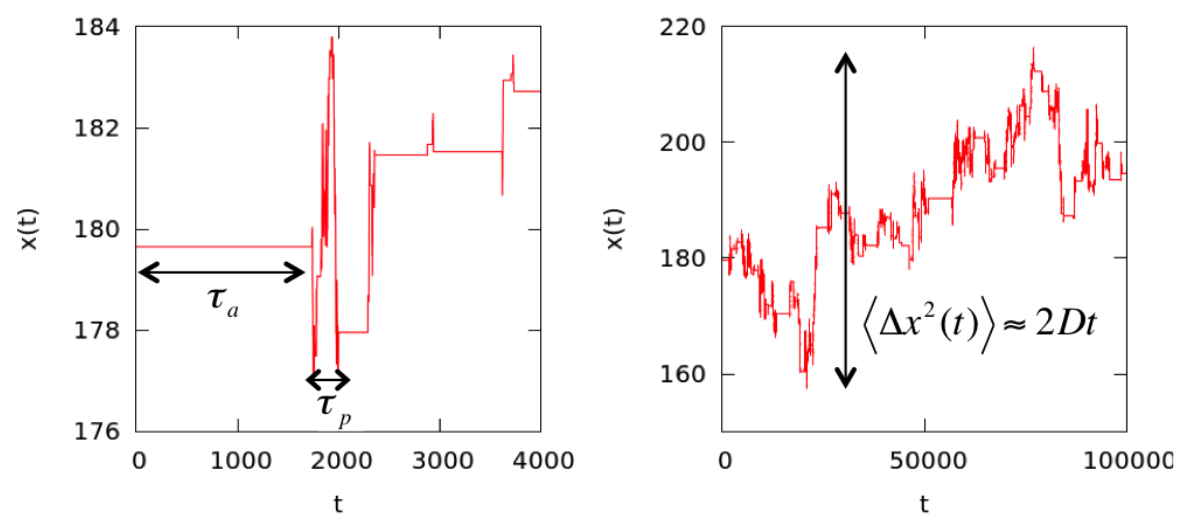

Figure 7. Typical trajectory of a single particle in the system (projected along the $x$-axis). The particle remains passive during some time interval characterised by $\tau_{a}$ and becomes mobile during another time interval characterised by $\tau_{p} \ll \tau_{a}$. If we look at the trajectory of the particle over larger times compared to $\tau_{a}$ and $\tau_{p}$, as in the right panel, the trajectory becomes typical of an ordinary random walker.

right panel of Fig. 7). Thus at large distances and times (as compared to the activation timescale), all particles are diffusive with diffusion constant $D$ :

$$
4 D=\lim _{t \rightarrow \infty} \frac{1}{t}\left\langle\frac{1}{N} \sum_{i=1}^{N}\left|\Delta \mathbf{r}_{i}(t)\right|^{2}\right\rangle,
$$

where $\Delta \mathbf{r}_{i}(t)=\mathbf{r}_{i}\left(t^{\prime}+t\right)-\mathbf{r}_{i}\left(t^{\prime}\right)$ with $t^{\prime}$ larger than the steady state time $t^{\prime}>t_{s s}$. The angle bracket indicates time average over many initial times $t^{\prime}$ as before. Note that the diffusion constant is a single particle statistics. Naturally, we expect the diffusion constant to be zero in the passive phase since the number of overlaps (hence mobile or active particles) goes to zero at steady state. On the other hand, the diffusion constant is finite in the active phase, i.e.

$$
D(\phi)=0, \phi<\phi_{c}, \quad D(\phi)>0, \phi>\phi_{c} .
$$

Moreover, it can be shown that $D$ is proportional to the order parameter/fraction of active particles in the system: $D \propto\left\langle f_{a}\right\rangle$. To see this, we observe that

$$
\Delta \mathbf{r}_{i}(t)=\int_{t^{\prime}}^{t^{\prime}+t} d t^{\prime \prime} f_{i}\left(t^{\prime \prime}\right) \vec{\delta}_{i}\left(t^{\prime \prime}\right),
$$

where $\vec{\delta}_{i}$ is the random displacement that we give to particle $i$ (but only if particle $i$ is active, i.e. $\left.f_{i}=1\right)$. From Eq. 40$\rangle$, it follows that $\left|\Delta \mathbf{r}_{i}(t)\right|^{2}=\left\langle f_{a}\right\rangle\left\langle\vec{\delta}_{i}^{2}\right\rangle t$ and thus:

$$
D=\left\langle f_{a}\right\rangle D_{a},
$$

where $D_{a}=\left\langle\vec{\delta}_{i}^{2}\right\rangle / 4$ is the diffusion constant that an (hypothetical) always-active particle would have. Finally since $D \propto\left\langle f_{a}\right\rangle$, the diffusion constant vanishes continuously at critical point with the same critical exponent as that of the order parameter $\left(D \sim\left(\phi-\phi_{c}\right)^{\beta}\right.$ with $\left.\beta \simeq 0.59\right)$. Effectively, the diffusion constant does not give us new information but experimental measurement of $D(\phi)$ can be useful to infer $\left\langle f_{a}\right\rangle(\phi)$. 


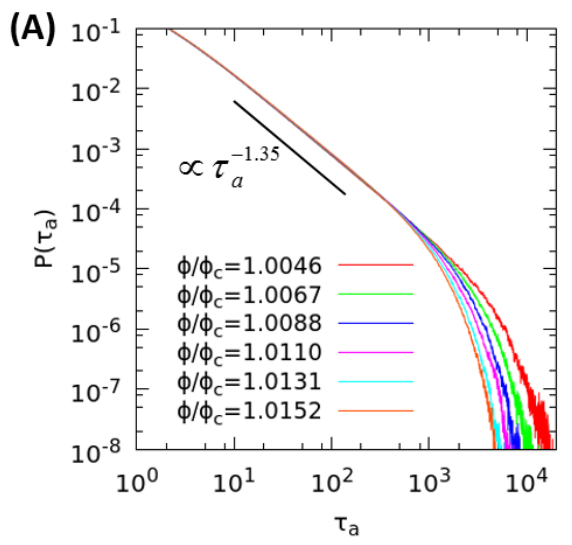

(B)
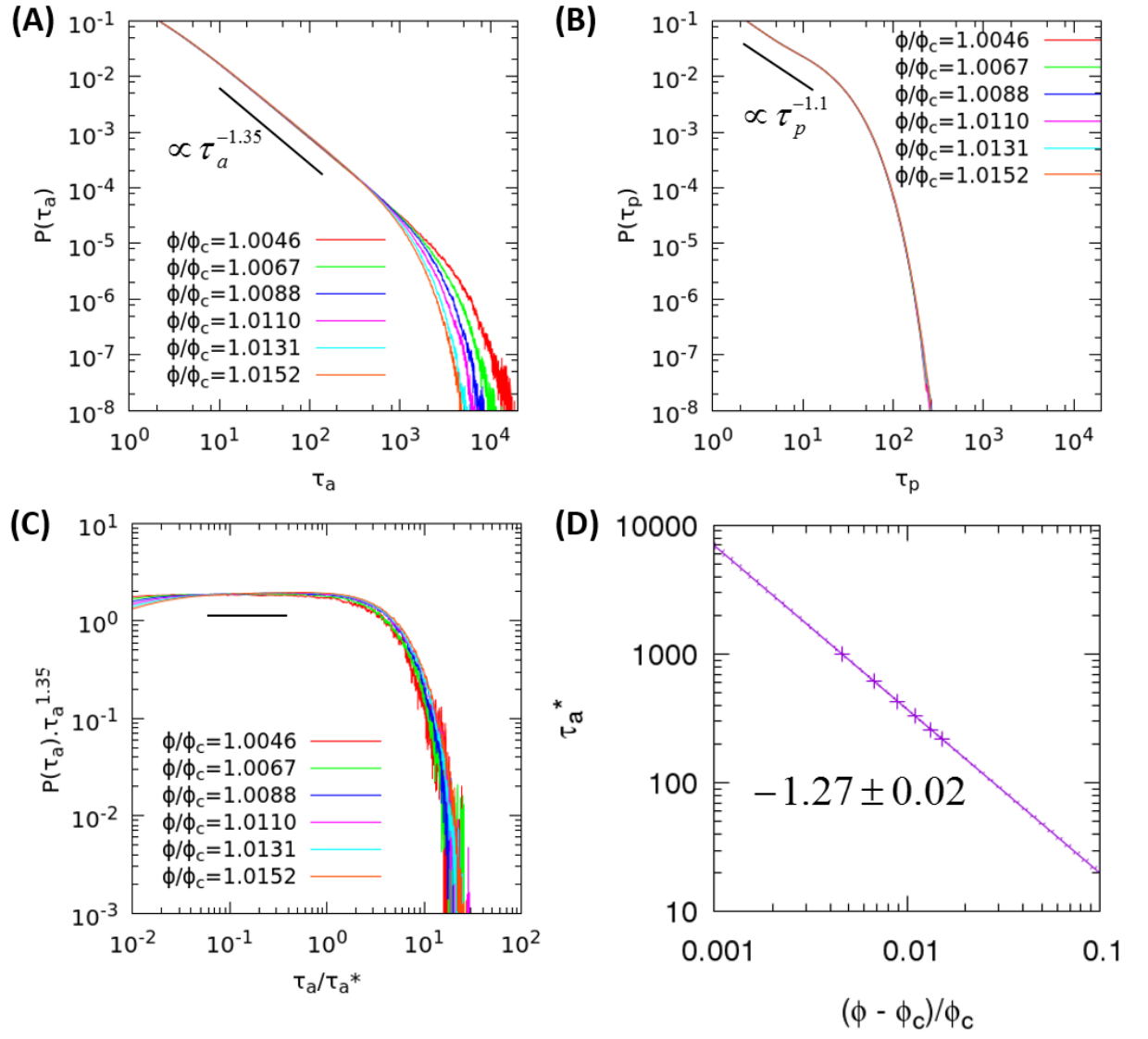

Figure 8. (A) The distribution of the activation timescale $P\left(\tau_{a}\right)$ exhibits a power law behaviour at short times and an exponential cut-off at large times: $P\left(\tau_{a}\right) \sim$ $\tau_{a}^{-z} \exp \left(-\tau_{a} / \tau_{a}^{*}(\phi)\right)$ with $z=1.35$ and an increasing timescale $\tau_{a}^{*}(\phi)$ as $\phi \rightarrow \phi_{c}^{+}$. (B) The distribution of the passivation timescale $P\left(\tau_{p}\right)$ also exhibits a power law behaviour with an exponential cut-off. Contrary to $P\left(\tau_{a}\right), P\left(\tau_{p}\right)$ does not depend on the density. (C) To find $\tau_{a}^{*}(\phi)$, we collapse $\tau_{a}^{z} P\left(\tau_{a}\right)$ against $\tau_{a} / \tau_{a}^{*}(\phi)$. (D) The characteristic timescale $\tau_{a}^{*}(\phi)$ diverges as $\tau_{a}^{*} \sim\left(\phi-\phi_{c}\right)^{-1.25}$.

Figs. 8(A,B) show the distribution of the activation and passivation timescales. As can be seen from the logarithmic scale, both distributions exhibit a power law behaviour at short times with an exponential cut-off at large times, i.e.

$$
P(\tau) \sim \tau^{-z} e^{-\tau / \tau^{*}(\phi)}
$$

In the case of the distribution of activation timescales $P\left(\tau_{a}\right)$, the exponential tail becomes longer as we approach the critical point (see Fig. 8(A)). On the other hand, the probability distribution of the passivation timescales $P\left(\tau_{p}\right)$ does not change with the packing fraction $\phi$ since $\tau_{p}$ only depends on the size of the random kicks $\delta$ that we give to the active particles (see Fig. 8(B)). Furthermore at steady state, we have $\left\langle f_{a}\right\rangle\left\langle\tau_{a}\right\rangle=\left(1-\left\langle f_{a}\right\rangle\right)\left\langle\tau_{p}\right\rangle$ due to equal flux of passive to active and passive to active conversion, where $\left\langle\tau_{a, p}\right\rangle$ is the average of $\tau_{a, p}$ over the probability distribution $P\left(\tau_{a, p}\right)$.

We can characterise the exponential cutoff by some relaxation timescale $\tau_{a}^{*}$ according to Eq. 42). In the case of the $P\left(\tau_{a}\right)$, this time scale $\tau_{a}^{*}$ grows as we approach 
the critical density $\phi \rightarrow \phi_{c}^{+}$. To see this, we plot $\tau_{a}^{z} P\left(\tau_{a}\right)$ as a function of $\tau_{a} / \tau_{a}^{*}(\phi)$ such that all plots for different $\phi$ 's collapse into a single curve at large $\tau_{a}$ (see Fig. $8(\mathrm{C})$ ). From data collapse, we obtain $\tau_{a}^{*}$ as a function of the packing fraction $\phi$ which indeed diverges at criticality with a power law behaviour: $\tau_{a}^{*} \sim\left(\phi-\phi_{c}\right)^{-\nu_{\|}}$as $\phi \rightarrow \phi_{c}^{+}$with critical exponent $\nu_{\|} \simeq 1.25$ (see Fig. 8(D)). Below we shall look at how the characteristic activation timescale $\tau_{a}^{*}$ may be probed experimentally.

Let us define $c(q, t)$ as:

$$
c(q, t)=\frac{1}{N} \sum_{i=1}^{N} e^{i \mathbf{q} \cdot \Delta \mathbf{r}_{i}(t)},
$$

where $\Delta \mathbf{r}_{i}(t)=\mathbf{r}_{i}\left(t^{\prime}+t\right)-\mathbf{r}_{i}\left(t^{\prime}\right)$ is the particle's displacement after time $t$. Physically, the quantity above tells us about the fraction of particles which have moved a distance $\lambda=\frac{2 \pi}{q}$ during the time interval $\left[t^{\prime}, t^{\prime}+t\right]$. The intermediate scattering function is defined to be the average of $c(q, t)$ over many initial times $t^{\prime}$ :

$$
F_{s}(q, t)=\langle c(q, t)\rangle .
$$

Experimentally, $F_{s}(q, t)$ is obtained from the intensity of monochromatic light scattered from the sample, or directly using microscopy techniques depending on the typical particle size considered. Fig. 9(A) shows the typical intermediate scattering function as a function of time $t$ for different wavevectors $q$. By equating $F_{s}(q, \tau(q))=e^{-1}$, we obtain a relaxation timescale $\tau(q)$ which depends on the wavevector $q$ (see Fig. 9(B)). From this figure, we see that $\tau(q)$ scales as $q^{-2}$ for small $q$ which is expected since in the limit of $q \rightarrow 0$ (long wavelengths and timescales limit), all particles behave like a random walker (see the trajectory in Fig. 7 (B) right). On the other hand for large $q \rightarrow \infty$, the relaxation time saturates to a finite value $\tau_{\infty}=\tau(q \rightarrow \infty)$. Thus from the intermediate scattering function, we may extract a characteristic timescale $\tau_{\infty}(\phi)$ which diverges as we approach criticality from above $\phi \rightarrow \phi_{c}^{+}$with scaling law: $\tau_{\infty} \sim\left(\phi-\phi_{c}\right)^{-\nu_{\|}}$and a critical exponent $\nu_{\|} \simeq 1.28$ (see Fig. $9(\mathrm{~B})$ ).

Physically, $\tau_{\infty}$ gives us the timescale for all particles to move from their original positions $\left\{\mathbf{r}_{i}\left(t^{\prime}\right)\right\}$. Thus, $\tau_{\infty}$ is also the waiting time for all particles to become active at some point during the time interval $\left[t^{\prime}, t^{\prime}+t\right]$. However, we also know that the waiting time $\tau_{\infty}$ is constrained by the probability distribution of the activation timescales $P\left(\tau_{a}\right)$. In particular, $\tau_{\infty}$ is limited by the long tail distribution of $P\left(\tau_{a}\right)$. From Fig. $8(\mathrm{C}), P\left(\tau_{a}\right)$ has an exponential tail as follows:

$$
P\left(\tau_{a}\right) \sim \exp \left(-\frac{\tau_{a}}{\tau_{a}^{*}(\phi)}\right)
$$

thus we deduce that $\tau_{\infty} \simeq \tau_{a}^{*}$. Indeed, both $\tau_{\infty}(\phi)$ and $\tau_{a}^{*}(\phi)$ diverge as $\phi \rightarrow \phi_{c}^{+}$with the same critical exponent ( $c f$. Fig. $8(\mathrm{D})$ to Fig. $9(\mathrm{C})$ ).

Finally, we may define a Fickian/diffusion lengthscale to be: $\ell_{F}=\sqrt{4 D \tau_{\infty}}$. This lengthscale is defined to be the crossover lengthscale from a Fickian $\left[\tau \sim 1 /\left(D q^{2}\right)\right]$ to a non-Fickian dynamics $\left[\tau \sim \tau_{\infty}(\phi)\right]$ (see Fig. 99(B)). From above, we know that the diffusion constant and the relaxation time scale as: $D \sim\left(\phi-\phi_{c}\right)^{0.59}$ and 

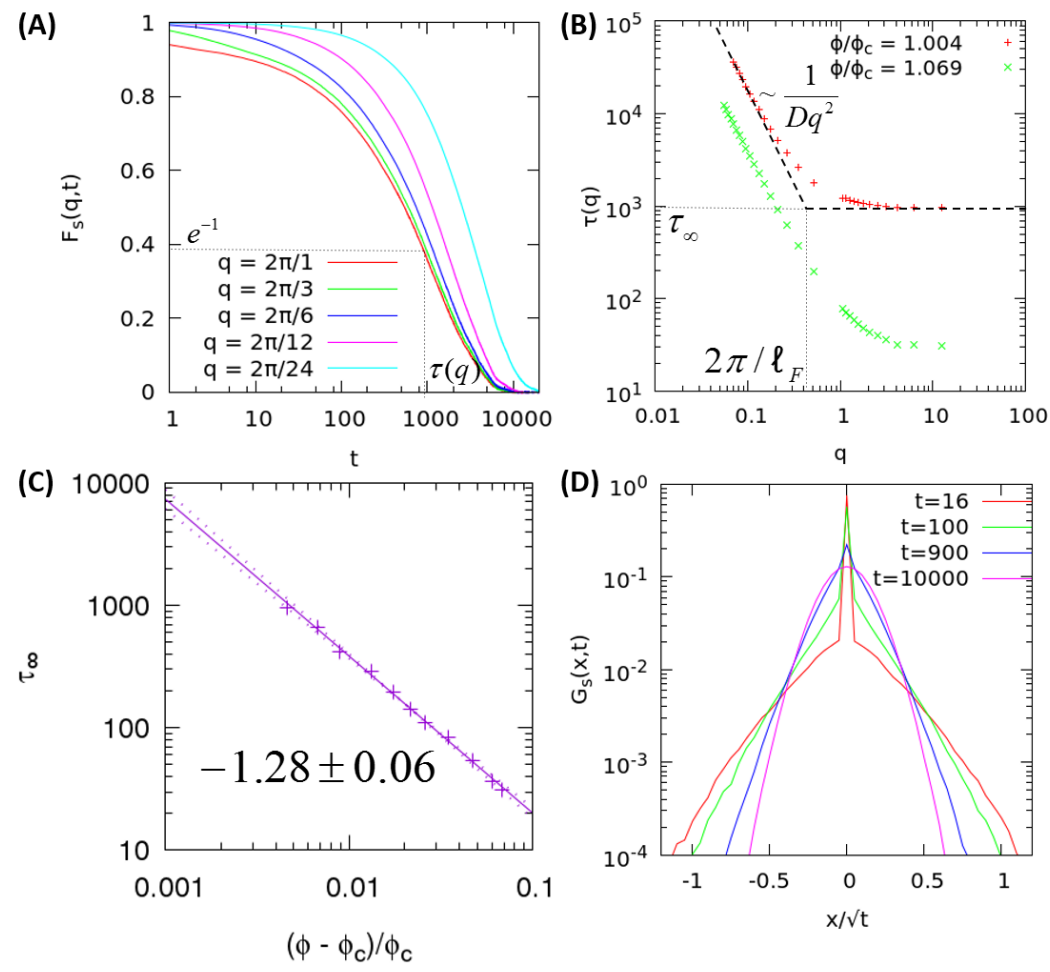

Figure 9. (A) Intermediate scattering function as a function of time $t$ for different wavectors $q$ for density $\phi / \phi_{c}=1.0044$. (B) The wave-vector dependence of relaxation time $\tau(q)$ for two different densities crosses over from diffusive when $q \rightarrow 0$, and becomes strongly non-Fickian and saturates to a constant value $\tau_{\infty}$ at large $q$. The crossover from Fickian to non-Fickian dynamics defines a Fickian lengthscale $\ell_{F}(\phi)$. (C) The timescale $\tau_{\infty}$ diverges at $\phi_{c}$ with critical exponent $\nu_{\|} \simeq 1.28$. (D) The distribution of particle displacements $G_{s}(x, t)$ displays a sharp peak at $x=0$ for small times and tails that are broader than a Gaussian. The distribution converges to a Gaussian distribution at large times.

$\tau_{\infty} \sim\left(\phi-\phi_{c}\right)^{-1.28}$ respectively. Thus the Fickian lengthscale should diverge as $\ell_{F} \sim\left(\phi-\phi_{c}\right)^{-0.35}$ as $\phi \rightarrow \phi_{c}^{+}$. Notice that it has a different critical exponent from the correlation length $\left(\xi \sim\left(\phi-\phi_{c}\right)^{-0.73}\right)$ which we obtained in Sec. 4.1 and Sec. 4.4 . This is as we should expect because the Fickian lengthscale is the average distance that a single particle diffuse (irrespective of other particles) during a correlation time. On the other hand the correlation length $\xi$ contains information about the collective behaviour (such as clustering). The independance between the Fickian lengthscale and other correlation lenghtscales is also found in supercooled liquids [42, 43].

To illustrate further the onset of Fickian dynamics, we can look at the distribution of the particles' displacements during the time interval $\left[t^{\prime}, t^{\prime}+t\right]$. More precisely, the van Hove function is defined to be:

$$
G_{s}(x, t)=\left\langle\frac{1}{N} \sum_{i=1}^{N} \delta\left(x-\Delta x_{i}(t)\right)\right\rangle,
$$

where $\Delta x_{i}(t)=x_{i}\left(t^{\prime}+t\right)-x_{i}\left(t^{\prime}\right)$ is the particle's displacement in the $x$-direction. The 
angle bracket $\langle$.$\rangle indicates time averaging over many initial times t^{\prime}$. Basically, this gives us the probability of finding a particle with displacement $x$ after time $t$ (note that since the system is isotropic, the probability distribution in the $x$-coordinate is the same as that in the $y$-coordinate). The van Hove function is plotted against $x / \sqrt{t}$ in Fig. 9 for different values of $t$. (Notice that $x$ is rescaled by $\sqrt{t}$ to fit curves at large $t$ which may become diffusive i.e. $x \sim \sqrt{t}$.) At short times $t \sim \tau$, we see a sharp peak at $x=0$, indicating that a large fraction of particles have not moved (i.e. remained passive during $\left.\left[t^{\prime}, t^{\prime}+t\right]\right)$. As $t$ increases, the peak gradually decreases as more particles become active and diplaced from their original positions $\left\{x_{i}\left(t^{\prime}\right)\right\}$. Finally at long times $t \gg \tau$, all particles become diffusive and the van Hove function becomes Gaussian with a width $\propto \sqrt{t}$. The coexistence at short times of a large number of immobile particles with a small number of mobile ones is natural in the context of the transition to an absorbing state, because the concentration of active particles vanishes continuously as $\phi \rightarrow \phi_{c}^{+}$. This is also an important dynamic signature in systems near a glass transition [30, where mobility becomes sparse as the glass transition is approached.

\subsection{Collective dynamic heterogeneities}

In Sec. 4.1, we discussed how we may obtain a static correlation length in our sytem by looking at the distribution of the active and passive particles. By static, we mean that the activity of the particles is determined from one period of oscillation. In this section, we shall demonstrate how we can also obtain a dynamic lengthscale in our system. This means we are looking at the state/activity of the particles over time intervals larger than one period. To this end, we run the simulation for some time interval $\left[t^{\prime}, t^{\prime}+t\right]$ where the initial time $t^{\prime}$ is larger than the steady state time. During this time interval $\left[t^{\prime}, t^{\prime}+t\right]$ we identify particles which have been active at least once (in other words, particles which have moved from their original positions at $\left.\left\{\mathbf{r}_{i}\left(t^{\prime}\right)\right\}\right)$. We shall call these 'A' particles (red colour in Fig. 10). On the other hands, particles which remain passive or stay at the same positions during the whole time interval $\left[t^{\prime}, t^{\prime}+t\right]$ are labelled $\mathrm{P}$ particles (grey colour in the figure). Let us insist again that these labels are defined over a finite time interval and thus differ from the ' $a$ ' and ' $p$ ' labels used in Sec. 4 above.

As we can see from Fig. 10, we observe domains of A particles which grow with the time interval $t$, reminiscent of spinodal decomposition. Eventually, the system will be filled with only A particles as $t \rightarrow \infty$. Thus we may define a dynamic lengthscale $\xi_{4}(t)$ to be the correlation length between $\mathrm{A}$ and $\mathrm{P}$ particles at time $t$. In Fig. 10 , $\xi_{4}(t)$ represents the average distance between two clusters of A particles. Thus we expect the strength of dynamic correlations to increase initially before reaching a peak at $t=\tau_{4} *$ [30]. Following recipes devised in studies of supercooled liquids, the dynamic correlation length is defined to be: $\xi_{4}^{*}=\xi_{4}\left(\tau_{4}^{*}\right)$. Comparing the two systems at two different densities in Fig. 10, both $\tau_{4}^{*}$ and $\xi_{4}^{*}$ grow larger as we approach the critical density $\phi \rightarrow \phi_{c}^{+} \simeq 0.375$.

We now discuss how this dynamic lengthscale $\xi_{4}$ and timescale $\tau_{4}^{*}$ may be quantified. 

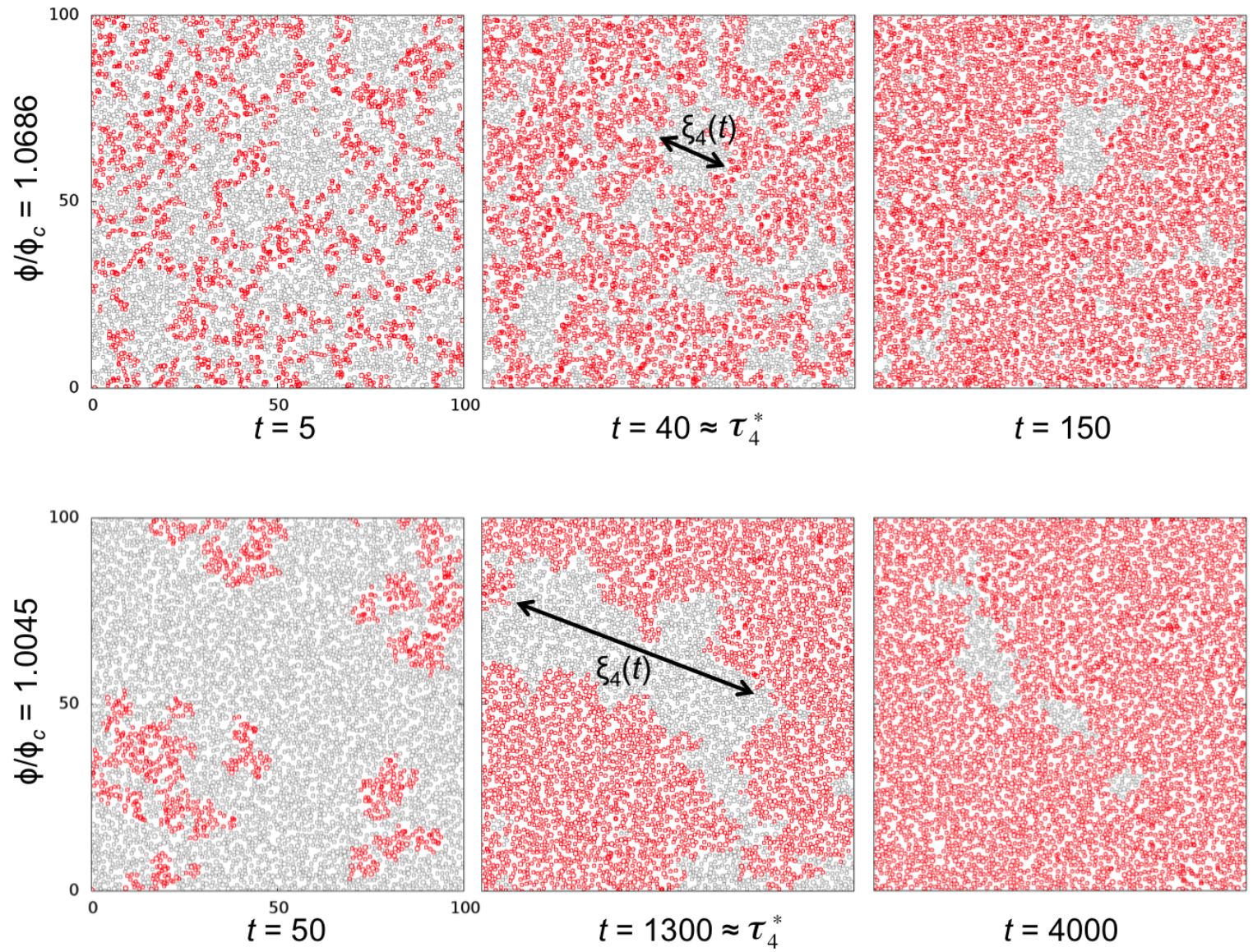

Figure 10. Time evolution of dynamic activity. Particles that have been mobile between the time interval $t$ are shown as red, always passive particles as grey. The correlation length between mobile and immobile particles defines a dynamic lengthscale $\xi_{4}(t)$. The dynamic lengthscale $\xi_{4}(t)$ increases with the time interval $t$ at fixed density, and also when approaching the critical point. Note that the snapshots only show a small portion of a larger system with $L=280$.

Recall the definition of the static structure factor $S(q)$ in Eq. 12 . Similarly, we may also define the dynamic structure factor to be [30]:

$$
S_{4}(k, t)=\left\langle\frac{1}{N} \sum_{i=1}^{N} \sum_{j=1}^{N} e^{i \mathbf{k} \cdot\left(\mathbf{r}_{i}-\mathbf{r}_{j}\right)} c_{i}(q, t)\left[c_{j}(q, t)-1\right]\right\rangle-\left\langle c_{i}\left(c_{i}-1\right)\right\rangle \delta_{\mathbf{k}, 0},
$$

where $c_{i}(q, t)$ is already defined in Eq. (43). For the rest of the discussion below, we shall set the wavector $q$ to be fixed at: $q=2 \pi$. (This value of $q$ corresponds to the saturation value of $\tau(q)$ in Fig. 9). Physically, $\left\langle c_{i}(q, t)\right\rangle=1$ if particle $i$ remains inactive during time interval $\left[t^{\prime}, t^{\prime}+t\right]$ and $\left\langle c_{i}(q, t)\right\rangle=0$ if particle $i$ has been active at least once during time interval $\left[t^{\prime}, t^{\prime}+t\right]$. Thus the dynamic structure factor defined above tells us about the correlation between $\mathrm{A}\left(\left\langle c_{i}(q, t)\right\rangle=0\right)$ and $\mathrm{P}$ particles $\left(\left\langle c_{i}(q, t)\right\rangle=1\right)$ in Fig. 10 at time $t$ (this is analogous to $S_{p a}(q)$ in the static case).

For completeness, we may also define the dynamic structure between pairs of A 

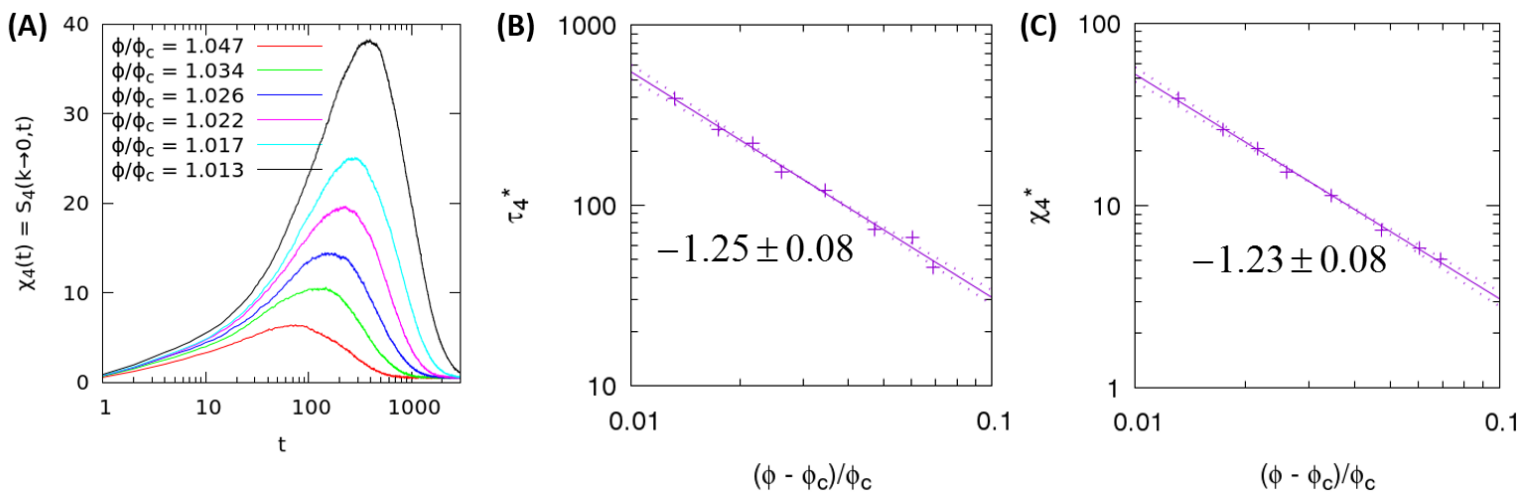

Figure 11. (A) Time dependence of the dynamic susceptibility $\chi_{4}(t)$ quantifying correlation between particles which have moved (A particles) and particles which are still at the same position (P particles) after time $t$. It shows a peak at $t=\tau_{4}^{*}(\phi)$, whose amplitude grows as $\phi \rightarrow \phi_{c}^{+}$. (B) The timescale $\tau_{4}^{*}$ diverges as $\tau_{4}^{*} \sim\left(\phi-\phi_{c}\right)^{-\nu_{\|}}$ where $\nu_{\|} \simeq 1.25$. (C) The maximum correlation $\chi_{4}^{*}$ also diverges at criticality, $\chi_{4}^{*} \sim\left(\phi-\phi_{c}\right)^{-\gamma^{\prime}}$ where $\gamma^{\prime} \simeq 1.23$.

or P particles, which are the dynamical analogs to the partial structure factors $S_{a a}(q)$ and $S_{p p}(q)$ in the static case. However since we are only interested in the maximum fluctuations (which occurs at $t=\tau_{4}^{*}$ ), the distribution of $\mathrm{A}$ and $\mathrm{P}$ particles are almost symmetric (see middle panels in Fig. 10). In fact we found that at $t=\tau_{4}^{*}$ and for small $k \ll 2 \pi / \xi_{4}$, all three partial dynamic structure factors are indeed similar. Thus for the rest of the discussion below, we shall only consider the mixed dynamic structure factor $S_{4}(k, t)$ as defined in Eq. (47).

We also define the corresponding dynamic susceptibility to be:

$$
\chi_{4}(t)=S_{4}(k \rightarrow 0, t)=\frac{\sqrt{\Delta N_{\mathrm{A}}(t) \Delta N_{\mathrm{P}}(t)}}{N_{\mathrm{A}}(t) N_{\mathrm{P}}(t)},
$$

which is the mixed number density fluctuation for $\mathrm{A}$ and $\mathrm{P}$ particles, analogous to the static case in Eq. (15) from Sec. 4.1. In other words, $\chi_{4}(t)$ measures the correlation between particles which have moved and particles which are still at the same positions after time $t$. The dynamic susceptibility is plotted in Fig. 11(A) for different densities $\phi$. As we can see from Fig. 11(A), $\chi_{4}(t)$ initially increases with the time interval $t$ and it reaches a peak at $t=\tau_{4}^{*}$ before decreases to zero as $t \rightarrow \infty$. We define the maximum correlation between $\mathrm{A}$ and $\mathrm{P}$ particles to be: $\chi_{4}^{*}=\chi_{4}\left(\tau_{4}^{*}\right)$. Both $\tau_{4}^{*}(\phi)$ and $\chi_{4}^{*}(\phi)$ are plotted in Fig. 11(B) and (C) respectively and shown to diverge as $\phi \rightarrow \phi_{c}^{+}$with critical exponents indicated in the figure. In particular, $\tau_{4}^{*}$ has a very similar critical exponent $\left(\nu_{\|} \simeq 1.24\right)$ to three other timescales that we have calculated earlier in this paper.

Note that alternatively, we may also define the dynamic susceptibility to be the fluctuations squared of $c(q, t)$ :

$$
\chi_{4}^{c}(t)=N\left\langle\Delta c^{2}(q, t)\right\rangle,
$$



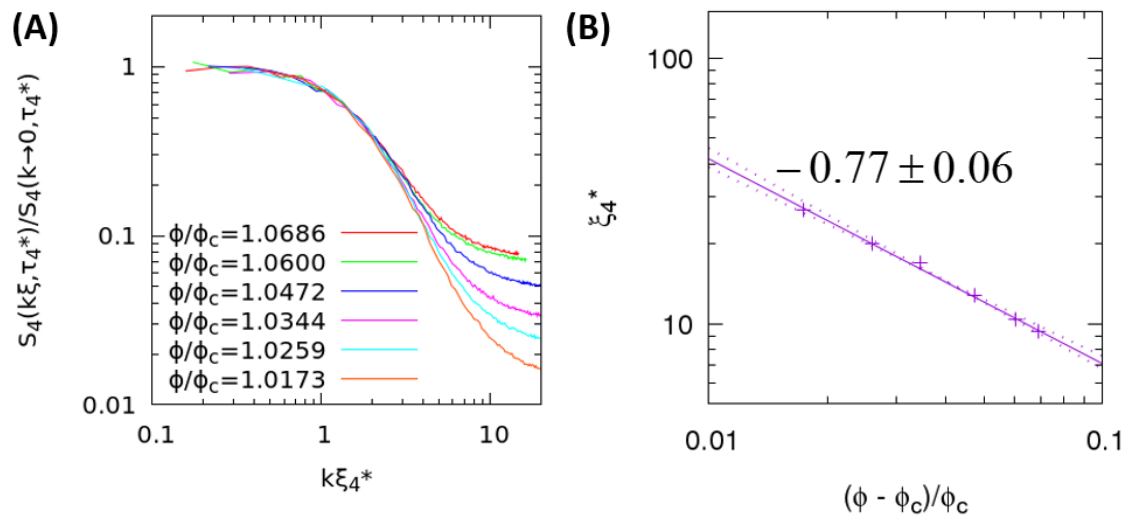

Figure 12. (A) Dynamic structure factor $S_{4}\left(k, t=\tau_{4}^{*}\right)$ as a function of the wavevector $k$ for different densities. We rescale $S_{4}\left(k, \tau_{4}^{*}\right)$ as in Eq. (50) to extract the dynamic lengthscale $\xi_{4}^{*}(\phi)$. (B) The dynamic lengthscale $\xi_{4}^{*}(\phi)$ diverges as $\xi_{4}^{*} \sim\left(\phi-\phi_{c}\right)^{-\nu_{\perp}}$ where $\nu_{\perp} \simeq 0.77$.

where $q=\frac{2 \pi}{\sigma}$. Using this definition, we also found that the timescale for $\chi_{4}^{c}(t)$ to reach its peak value $\left(\tau_{4}^{c *}\right)$ diverges with the same critical exponent as $\tau_{4}^{*}$. However the peak height $\chi_{4}^{c *}$ does not diverge with the same critical exponent as $\chi_{4}^{*}$ due to the difference between canonical and grand-canonical ensemble when calculating $\chi_{4}^{c}(t)$ and $\chi_{4}(t)(c f$. Sec. 4.2.

Finally to calculate the dynamic lengthscale $\xi_{4}(t)$, we fit the dynamic structure factor $S_{4}(k, t)$ into the following scaling form (similar to the static case in Eq. (34)):

$$
\frac{S_{4}(k, t ; \phi)}{S_{4}(k \rightarrow 0, t ; \phi)}=F\left(k \xi_{4}(t ; \phi)\right), \quad k \ll \frac{2 \pi}{\xi_{4}},
$$

where $F(x)$ is some universal function independent of $\phi$. Since we are only interested in the maximum dynamic lengthscale $\xi_{4}^{*}$, we shall fix $t=\tau_{4}^{*}$. In Fig. 12, we plot $S_{4}\left(k, \tau_{4}^{*}\right) / S_{4}\left(k \rightarrow 0, \tau_{4}^{*}\right)$ as a function of $k \xi_{4}^{*}$ such that all curves corresponding to different $\phi$ 's collapse into a single function: $F\left(k \xi_{4}^{*}\right)$ at small $k$. From this plot, we extract the maximum dynamic lengthscale $\xi_{4}^{*}(\phi)$ which again diverges at the critical density with a power law: $\xi_{4}^{*} \sim\left(\phi-\phi_{c}\right)^{-\nu_{\perp}}$ as $\phi \rightarrow \phi_{c}^{+}$. The critical exponent $\nu_{\perp} \simeq 0.77$, in this case, is similar to that found from the static lengthscales. Thus close to criticality, both dynamic and static lengthscales diverge with the same critical exponent, differing only in their prefactors.

\section{Conclusions}

In conclusion, we have introduced and studied numerically a simple and generic model of periodically driven suspensions which possesses a non-equilibrium absorbing phase transition at a critical density $\phi_{c}$. In particular, our model has provided a closer connection to (conserved) directed percolation universality classes by removing the anisotropy associated with sheared suspensions, which might otherwise affect the critical 


\begin{tabular}{c|ccc} 
quantities & our model & DP & CDP \\
\hline order parameter, $\left\langle f_{a}\right\rangle \sim\left(\phi-\phi_{c}\right)^{\beta}$ & $0.59 \pm 0.02$ & 0.58 & 0.64 \\
order parameter decay, $f_{a}(t) \sim t^{-\alpha}$ at $\phi=\phi_{c}$ & 0.45 & 0.45 & 0.42 \\
fluctuations of the order parameter, $\left\langle N \Delta f_{a}^{2}\right\rangle_{g c} \sim\left(\phi-\phi_{c}\right)^{-\gamma_{g c}}$ & $0.32 \pm 0.02$ & 0.30 & 0.37 \\
timescale, $\tau \sim\left(\phi-\phi_{c}\right)^{-\nu_{\|}}$ & $1.26 \pm 0.03$ & 1.30 & 1.23 \\
lengthscale, $\xi \sim\left(\phi-\phi_{c}\right)^{-\nu_{\perp}}$ & $0.74 \pm 0.03$ & 0.73 & 0.80
\end{tabular}

Table 1. Critical exponents of the order parameter $\left(\left\langle f_{a}\right\rangle, f_{a}(t)\right)$, fluctuation squared of the order parameter $\left(\left\langle N \Delta^{2} f_{a}\right\rangle\right)$, timescales $(\tau)$ and lengthscales $(\xi)$ as compared to directed percolation (DP) and conserved directed percolation (CDP) [2, 3].

behaviour observed numerically by introducing a short-scale anisotropic behaviour of physical quantities 32 . In this paper, both static and dynamic correlations lengths and associated susceptibilities are also measured for the first time. In particular, the dynamic correlation length is obtained from dynamic heterogeneity in both space and time, in analogy to glassy systems [30]. Both static and dynamic lengthscales produce similar critical exponent $\left(\nu_{\perp}\right)$. We also measured several different timescales independently and they are all shown to diverge again with a similar critical exponent $\left(\nu_{\|}\right)$. Our main achievement has been the demonstration that a large number of physical observables, commonly measured in experimental work on colloidal suspensions, provide direct access to the criticality associated to the non-equilibrium phase transition. Thus, the mere existence of such a phase transition in experimental work dealing with real suspensions subjected to an oscillatory shear flow is a question that should be amenable to decisive experimental investigations guided by the present results.

In this respect, we have established a useful analogy with the physics of glassy systems such as intermittent dynamics and spatially heterogeneous dynamical relaxation. The analogy with such materials is not only a curiosity. It also provides a theoretical framework and practical tools to characterize the relevant physical observables characterizing the dynamics in systems close to a non-equilibrium phase transition, despite the distinct physical origin of dynamic correlations in both types of systems. Our model also shows true hyperuniformity at large enough lengthscales, with an exponent $\lambda=1$ at criticality $\phi_{c}$. The issue of hyperuniformity was tackled in several recent studies [9, 21, 28, 29].

The question of the universality class of the transition found in the present model is a more difficult problem, as the naturally expected types of transitions for this system, directed percolation and conserved directed percolation, are characterized by very close sets of critical exponents and no real qualitative difference between them [2, 3]. Our measurements for all these critical exponents are summarised in Table 1 and for comparison, critical exponents for the directed percolation (DP) and conserved directed percolation (CDP) universality class are also shown on the table. Given how close the two sets of exponents are, it is no surprise that our measured exponents remain quantitatively consistent with both DP or CDP universality classes, despite 
our large numerical effort. We found for instance that our results are robust against finite size effects and extensive time averaging in very large systems. More careful determination of critical exponents seeking to make a decisive statement about the DP versus CDP universality class from a purely computational perspective would have to involve a truly significant computational effort, perhaps focusing on only a handlful of the measurements reported in our work.

At present, it would seem that our critical exponents favor slightly the DP universality class, as can be judged by the values reported in Table 1. This is a little surprising at first sight, as the main physical ingredient identified in lattice models to distinguish between the two families of models is the presence of particle conservation in the microscopic rules defining the model [2, 3]. This remark forms the basis of the approach put forward in Ref. [23], which suggests that the present model should belong to the CDP universality class. How to reconcile our numerical findings with this natural expectations? A first possibility is that a crossover to CDP values of the critical exponents would occur only much closer to the critical point, for instance because the role of particle conservation is only effective extremely close to the critical point. In this view, we would interpret our measurements as being pre-asymptotic (despite our largest correlation length being of about 50 particle diameters) and, for reasons that remain to be elucidated, this regime appears closer to the DP behaviour. A second hypothesis is that our results reflect the true asymptotic behaviour of the model. This alternative view implies that despite the presence of particle conservation in the microscopic dynamics, its effect is not felt at large enough lengthscales close to the critical point; in other word the critical behaviour of the model is truly that of directed percolation and particle conservation is asymptotically irrelevant. As mentioned several times, we believe it will challenging to tell the two hypothesis apart on the basis of numerical simulations only, and progress should come from a theoretical treatment of the critical behaviour of the model, which remains to be performed.

In conclusion, we hope that our simplified model will open up new theoretical approaches in the area of periodically driven non-equilibrium systems, as well as motivate novel experiments to determine and expose the nature of the non-equilibrium phase transition seen in periodically sheared suspensions.

\section{Acknowledgments}

We thank D. Bartolo, J.-P. Bouchaud and R. Jack for useful discussions. The research leading to these results has received funding from the European Research Council under the European Unions Seventh Framework Programme (FP7/2007-2013)/ERC Grant Agreement No. 306845.

\section{References}

[1] H. Hinrichsen, Nonequilibrium critical phenomena and phase transitions into absorbing states, Adv. Phys. 49 (2000) 815. 
[2] S. Lubeck, Universal scaling behaviour of non-equilibrium phase-transitions, Int. J. Mod. Phys. B 18 (2004) 3977.

[3] M. Henkel, H. Hinrichsen, and S. Lubeck, Non-Equilibrium Phase Transitions Vol. 1. Absorbing Phase Transitions, Springer, 2008.

[4] D. J. Pine, J. P. Gollub, J. F. Brady, and A. M. Leshansky, Chaos and irreversibility in sheared suspensions, Nature 438 (2005) 997.

[5] L. Corté, P. M. Chaikin, J. P. Gollub, and D. J. Pine, Random organization in periodically driven systems, Nature Physics 4 (2008) 420.

[6] L. Corté, S. J. Gerbode, W. Man, and D. J. Pine, Self-Organized Criticality in Sheared Suspensions Phys. Rev. Lett. 103 (2009) 248301.

[7] R. Jeanneret and D. Bartolo, Geometrically protected reversibility in hydrodynamic Loschmidtecho experiments, Nat. Comm. 5 (2014) 3474.

[8] K. H. Nagamanasa, S. Gokhale, A. K. Sood, and R. Ganapathy, Experimental signatures of a nonequilibrium phase transition governing the yielding of a soft glass, Phys. Rev. E 89 (2014) 062308 .

[9] J. H. Weijs, R. J. Dreyfus, and D. Bartolo, Emergent hyperuniformity in periodically-driven emulsions, Phys. Rev. Lett. 114 (2015) 110602.

[10] A. Franceschini, E. Filippidi, E. Guazzelli, and D. J. Pine, Dynamics of non-Brownian fiber suspensions under periodic shead, Soft Matter 10 (2014) 6722.

[11] J. S. Guasto, A. S. Ross, and J. P. Gollub, Hydrodynamic irreversibility in particle suspensions with nonuniform strain, Phys. Rev. E 81 (2010) 061401.

[12] E. D. Knowlton, D. J. Pine, and L. Cipelletti, A microscopic view of the yielding transition in concentrated emulsions, Soft Matter 10 (2014) 6931.

[13] T. Kawasaki and L. Berthier, Macroscopic yielding in jammed solids is accompanied by a nonequilibrium first-order transition in particle trajectories, arXiv:1507.04120.

[14] N. C. Keim and P. E. Arratia, Mechanical and microscopic properties of the reversible plastic regime in a 2D jammed material, Phys. Rev. Lett. 112 (2014) 028302.

[15] B. Metzger and J. E. Butler, Irreversibility and chaos: Role of long-range hydrodynamic interactions in sheared suspensions, Phys. Rev. E 82 (2010) 051406.

[16] B. Metzger, P. Pham, and J. E. Butler, Irreversibility and chaos: Role of lubrication interactions in sheared suspensions, Phys. Rev. E 87 (2013) 052304.

[17] P. Sundararajan, J. D. Kirtland, D. L. Koch, and A. D. Stroock, Impact of chaos and Brownian diffusion on irreversibility in Stokes flows, Phys. Rev. E 86 (2012) 046203.

[18] G. Düring, D. Bartolo, and J. Kurchan, Irreversibility and self-organisation in hydrodynamic echo experiments, Phys. Rev. E 79 (2009) 030101.

[19] I. Regev, T. Lookman, and C. Reichhardt, Onset of irreversibility and chaos in amorphous solids under periodic shear, Phys. Rev. E 88 (2013) 062401.

[20] C. F. Schreck, R. S. Hoy, M. D. Shattuck, and C. S. O'Hern, Particle-scale reversibility in athermal particulate media below jamming, Phys. Rev. E 88 (2013) 052205.

[21] E. Tjhung and L. Berthier, Hyperuniform density fluctuations and diverging dynamic correlations in periodically driven colloidal suspensions, Phys. Rev. Lett. 114 (2015) 148301.

[22] L. Milz and M. Schmiedeberg, Connecting the random organization transition and jamming within a unifying model system, Phys. Rev. E 88 (2013) 062308.

[23] G. I. Menon and S. Ramaswamy, Universality class of the reversible-irreversible transition in sheared suspensions, Phys. Rev. E 79 (2009) 061108.

[24] N. Mangan, C. Reichhardt, and C. J. Olson Reichhardt, Reversible to irreversible flow transition in periodically driven vortices, Phys. Rev. Lett. 100 (2008) 187002.

[25] C. Reichhardt and C. J. Olson Reichhardt, Random organization and plastic depinning, Phys. Rev. Lett. 103 (2009) 168301.

[26] D. Fiocco, Oscillatory deformation of amorphous materials: a numerical investigation, $\mathrm{Ph} . \mathrm{D}$. Thesis. Ecole Polytechnique Federale de Lausanne, 2014. 
[27] D. Fiocco, G. Foffi, and S. Sastry, Oscillatory athermal quasistatic deformation of a model glass, Phys. Rev. E $8 \mathbf{8}$ (2013) 020301.

[28] D. Hexner and D. Levine, Hyperuniformity of critical absorbing states, Phys. Rev. Lett. 115 (2015) 108301.

[29] K. J. Schrenk and D. Frenkel, Evidence for non-ergodicity in quiescent states of periodically sheared suspensions, arXiv:1510.01280.

[30] L. Berthier, G. Biroli, J.-P. Bouchaud, L. Cipelletti, W. van Saarloos, Dynamical heterogeneities in glasses, colloids and granular materials, Oxford University Press, 2011.

[31] L. Berthier and G. Biroli, Theoretical perspective on the glass transition and amorphous materials, Rev. Mod. Phys. 83 (2011) 587.

[32] D. V. Denisov, M. T. Dang, B. Struth, G. H. Wegdam, and P. Schall, Particle response during the yielding transition of colloidal glasses, arXiv:1401.2106 v1.

[33] B. Percier, T. Divoux, and N. Taberlet, Insights on the local dynamics induced by thermal cycling in granular matter, Europhys. Lett. 104 (2013) 24001.

[34] P. J. Yunker, K. Chen, M. D. Gratale, M. A. Lohr, T. Still, and A. G. Yodh, Physics in ordered and disordered colloidal matter composed of poly(N-isopropylacrylamide) microgel particles, Rep. Prog. Phys. 77 (2014) 056601.

[35] J. L. Lebowitz, J. K. Percus, and L. Verlet, Ensemble Dependence of Fluctuations with Application to Machine Computations, Phys. Rev. 153, 250 (1967).

[36] A. B. Bhatia and D. E. Thornton, Structural Aspects of the Electrical Resistivity of Binary Alloys, Phys. Rev. B 2 (1970) 3004.

[37] S. Torquato and F. H. Stillinger, Local density fluctuations, hyperuniformity, and order metrics, Phys. Rev. E 68 (2003) 041113.

[38] A. Donev, F. H. Stillinger, and S. Torquato, Unexpected fluctuations in jammed disordered sphere packings, Phys. Rev. Lett. 95 (2005) 090604.

[39] L. Berthier, P. Chaudhuri, C. Coulais, O. Dauchot, and P. Sollich, Suppressed compressibility at large scale in jammed packings of size-disperse spheres, Phys. Rev. Lett. 106 (2011) 120601.

[40] A. Ikeda and L. Berthier, Thermal fluctuations, mechanical response, and hyperuniformity in jammed solids. Phys. Rev. E 92 (2015) 012309.

[41] Y. Wu, P. Olsson, and S. Teitel, Search for hyper uniformity in mechanically stable packings of frictionless disks above jamming, arXiv:1506.01948

[42] L. Berthier, D. Chandler, and J. P. Garrahan, Length scale for the onset of Fickian diffusion in supercooled liquids, Europhys. Lett. 71 (2005) 320.

[43] L. Berthier, Time and length scales in supercooled liquids, Phys. Rev. E 69 (2004) 020201(R). 